\title{
Electron, pion and multiparticle detection with a lead/scintillating- fiber calorimeter
}

\author{
D. Acosta ${ }^{\text {a }}$, S. Buontempo ${ }^{\text {b }}$, L. Calôba ${ }^{\text {c }}$, M. Caria ${ }^{\text {d }}$, R. DeSalvo ${ }^{\text {f }}$, A. Ereditato ${ }^{\text {, }}$, \\ R. Ferrari h, G. Fumagalli h, G. Goggi e,h, W. Hao g,1, F.G. Hartjes ', A. Henriques e, , \\ P. Jenni ${ }^{\text {e }}$, L. Linssen ${ }^{\mathrm{f}}$, M. Livan ${ }^{\mathrm{d}}$, A. Maio ', L. Mapelli e, M.R. Mondardini ${ }^{\mathrm{k}}$, B. Ong ${ }^{\mathrm{a}}$, \\ H.P. Paar ${ }^{\text {a }}$, F. Pastore ${ }^{\text {h }}$, L. Poggioli ', G. Polesello ${ }^{\text {e,h }}$, F. Riccardi ${ }^{\text {b }}$, A. Rimoldi ${ }^{\text {h }}$, \\ C.V. Scheel ${ }^{\text {f,i }}$, J. Schmitz ', J.M. Seixas ${ }^{\text {c,e }}$, A. Simon ${ }^{m}$, M. Sivertz a , P. Sonderegger e, \\ M.N. Souza ${ }^{\text {c }}$, Z.D. Thomé c , V. Vercesi h, Y. Wang ${ }^{\text {g, }}$, R. Wigmans ${ }^{\text {e,i }}$, C. Xu ${ }^{\text {g, } 1}$ \\ and $\mathrm{K}$. You $\mathrm{g}, 1$ \\ ${ }^{a}$ Unversity of Caltfornta, San Dtego, USA \\ ${ }^{b}$ Unicersità dı Napoli and INFN Sez. Napoll, Italy \\ ' COPPE / EE /UFRJ, Rio de Janeiro, Brazll \\ ${ }^{d}$ Università dl Cagliari and INFN Sez. Caglıari, Italy \\ ${ }^{e}$ CERN, Geneva, Switzerland \\ ${ }^{f}$ CERN / LAA project, Geneva, Swtzerland \\ ${ }^{g}$ CERN (Worldlab fellow), Geneva, Switzerland \\ ${ }^{h}$ Università dl Pavia and INFN Sez. Pavia, Italy \\ 'NIKHEF-H, Amsterdam, The Netherlands \\ ${ }^{\prime}$ LIP, Lisbon, Portugal \\ ${ }^{k}$ Cornell Unversity, Ithaca, USA \\ ${ }^{\prime}$ LPNHE, Unwersité Paris VI \& VII, Paris, France \\ ${ }^{m}$ Unuersitat Heidelberg, Heidelberg, Germany
}

Received 22 May 1991

We report on the performance of a fine-grained 13-ton lead/scintllating-fiber calorimeter, in particular on its response to electrons, pions and multiparticles (reaction products from pions interacting in a target upstream of the detector). The detector signals were studied for particles in the energy range $5-150 \mathrm{GeV}$. The energy resolution was measured to be $12.9 \% / \sqrt{E}$ for electrons, plus a constant term dependent on the angle $\theta_{z}$ between the particle's direction and the fiber axis. This term, which is $1.2 \%$ for $\theta_{z}=3^{\circ}$, is shown to be due to anomalous sampling in the early shower stage. It is greatly reduced when only electrons entering the detector in the lead are considered. A $1.7 X_{0}$ thick preshower detector, installed $12 \mathrm{~cm}$ in front of the calorimeter, only affected the signal linearity for electrons at low energy. The effect on the energy resolution was negligible.

Single pions were detected with an energy resolution of $\sim 30 \% / \sqrt{E}$ plus a constant term, which turned out to be mainly due to the effects of light attenuation in the fibers. Knowing the impact point of the particles, these effects could be efficiently removed for single pions. For jets (multiparticles), the effects of light attenuation are much less important, leading to considerably better on-line energy resolutions. The e/ $\pi$ signal ratio was measured to range from 1.03 at $80 \mathrm{GeV}$ to 1.10 at $5 \mathrm{GeV}$, for a detector with an effective radius of $49 \mathrm{~cm}$. After correcting for the instrumental effects, we found the intrinsic $e / h$ value of this detector (with our particular choice of fibers and sampling fraction) to be $1.15 \pm 0.02$. Detailed results are given on the detector performance (energy resolution, e/ $\pi$ signal ratio, e/jet signal ratio) as a function of the lateral detector size and as a function of the jet multiplicity.

\section{Introduction}

The advent of the new generation of multi-TeV high-luminosity pp colliders (LHC/SSC) presents a

1 On leave of absence from IHEP Beijing, China. challenge for developing detectors that can fruitfully operate in experiments with these machines. Calorimeters will play a central role in such experiments, since they have to provide the first-level trigger information. The calorimeter information is the first filter for selecting the rare interesting events under extreme back- 
ground conditions. Moreover, the calorimeter data is the basis for studying the production of jets and leptons, the likely messengers of new physics.

Precise energy measurements are a prime task of detectors in particle physics experiments. New unstable particles were always discovered from a kinematical reconstruction of their decay products, the quality of which is closely linked to the precision of the particle energy measurements. Therefore, the energy resolution of calorimeters in LHC/SSC experiments is an important issue, especially since also the background from conventional processes to most of the envisaged new physics signals is expected to be very large.

Good e.m.-energy resolution is considered to be important in view of the process $\mathrm{H}^{0} \rightarrow \gamma \gamma$, which is believed to be one of the most promising channels for detecting the Higgs particle if $m_{\mathrm{W}}<m_{\mathrm{H}}<2 m_{\mathrm{W}}$ [1]. The hadronic resolution is important for the precision of multi-jet spectroscopy and the missing (transverse) energy measurements.

The best energy resolutions for hadron detection are achieved with compensating calorimeters, i.e. detectors that respond with the same signal to the e.m. and non-e.m. components of hadronic showers (the $e / h=1$ condition). If the response to these components is different, the large and non-Gaussian fluctuations in the e.m. shower fraction will contribute to the energy resolution and dominate it at high energies [2]. There are, however other probably even more compelling reasons why $\mathrm{LHC} / \mathrm{SSC}$ experiments should preferably be equipped with compensating calorimeters. For example, the very steeply falling experimental distributions of quantities on which one will want to trigger ( $E_{\perp}^{\mathrm{jets}}, E_{\perp}^{\mathrm{m} i s s}$, etc.) will cause any event sample selected with the calorimeter data to be strongly biased with events containing an anomalous fraction of $\pi^{0}$ 's, if the $e / h$ ratio deviates from 1 . In view of the sensitivity of the trigger to errors in the energy measurement, it is important to have a first level trigger signal that is a direct measure of the particle energies, independent of the particle type.

In 1987, it was predicted [3] and proven experimentally [4] that the compensation condition could be fulfilled for a lead/plastic-scintillator sampling calorimeter. In order to achieve that goal, such a calorimeter should have a sampling fraction that is much smaller than usual, around $2 \%$. Bernardi et al. measured $\mathrm{e} / \pi$ signal ratios of $1.05 \pm 0.04$ at energies from 3-75 GeV, for a calorimeter consisting of $10 \mathrm{~mm}$ thick lead slabs alternating with $2.5 \mathrm{~mm}$ thick plastic scintillator plates [4]. The energy resolution $\sigma / E$ measured with this calorimeter amounted to $24 \% / \sqrt{E}$ for electrons and $45 \% / \sqrt{E}$ for pions. The same authors also measured the contribution of the soscalled intrinsic fluctuations (fluctuations in the fraction of the energy converted into a measurable form) to the latter result and found $(13 \pm 4) \% / \sqrt{E}[5,6]$, thus proving that the energy resolution of this calorimeter was largely dominated by the contribution of sampling fluctuations. Therefore, an improvement of the energy resolution may be expected by reducing the effect of these fluctuations.

Since the small sampling fraction is imposed by the compensation requirement, the only way to reduce the effect of sampling fluctuations is by increasing the sampling frequency of the showers. This can be achieved by using scintillating fibers instead of plates.

The Spaghetti Calorimeter (SPACAL) was developed, in the framework of the LAA project at CERN, as a detector intended to optimize compensating lead/plastic-scintillator calorimetry, with application in an LHC/SSC experiment in mind. It is a longitudinally unsegmented $\mathrm{Pb}$ /scintillating-fiber calorimeter intended for the detection of electromagnetic and hadronic showers, muons and missing energy.

Among its remarkable properties, we mention two that are particularly relevant for its performance in an LHC / SSC environment, namely the very high signal speed $[7,8]$ and the hermeticity. The latter is achieved because the fibers that sample the showers are running (approximately) in the same direction as the incoming particles, so that all the readout is located at the back of the detector [9]. Also the position resolution and the particle-particle separation, which together with the energy resolution determine the precision with which the mass of eventual new particles can be measured, were found to be very good, due to the fine lateral granularity and the high effective density of the detector $[10]$.

In this article, we discuss the performance of this calorimeter concerning the detection of electrons, single pions and "jets". The latter were simulated by the reaction products of pions interacting in a target placed upstream of the detector. In section 2 , the calorimeter and the tests in which the experimental data were obtained are described. In section 3 we discuss the experimental data and the methods used for analyzing them. The e.m. energy resolution is the subject of section 4. Experimental results concerning the hadronic energy resolution, the $\mathrm{e} / \pi$ signal ratios and the $e / h$ value are presented in section 5 for single pions, and in section 6 for multiparticle systems. The results are discussed in section 7 and summarized in section 8 .

\section{Experimental setup}

\subsection{The detector}

The measurements were performed with a calorimeter consisting of 155 longitudinally unsegmented towers. Each tower contains 1141 plastic scintillating 
fibers ${ }^{\# 1}$ with a diameter of $1 \mathrm{~mm}$ and a length of 2.20 $\mathrm{m}$.

These fibers form the active part of this sampling calorimeter. They are embedded in a lead matrix in such a way that each fiber is equidistant to its 6 neighbors (fig. 1a). The fiber spacing is $2.22 \mathrm{~mm}$ (center-to-center), such as to achieve a volume ratio lead: fiber of about $4: 1$, needed in order to make the calorimeter (approximately) compensating [3,4]. The detector was constructed by piling up extruded lead sheets with the appropriate profile (see fig. 1a).

The fibers were grouped to form 155 towers. Each tower has an hexagonal cross section $(86 \mathrm{~mm}$ apex-toapex). The depth of the lead structure is $200 \mathrm{~cm}$. The fibers sticking out at the back end of the tower were bunched together in an hexagonal structure, machined and polished and coupled through an hexagonal light guide ( $79 \mathrm{~mm}$ long, $42 \mathrm{~mm}$ apex-to-apex) to a photomultiplier (PM) \#2. The front ends of the fibers were polished and made reflective by aluminium sputtering, such as to make the response more uniform as a function of depth. More detailed information about the structure of the calorimeter can be found in ref. [9].

The lateral cross section of the detector as a whole is shown in fig. $1 \mathrm{~b}$. The central tower is surrounded by seven concentric hexagonal rings, with the outer ring incomplete. The detector has a roughly cylindrical shape with a diameter of 1 meter.

Because of the large fraction of high- $Z$ absorber material $77.7 \%$ in volume, with $18.4 \%$ plastic and $3.9 \%$ air), the shower dimensions are relatively small in this detector. The effective radiation length $\left(X_{0}\right)$

\#1 SCSF-38, produced by Kyowa Gas, now Kuraray Co. Ltd, Tokyo, Japan.

\#2 Philips XP 2282, 8-stage.
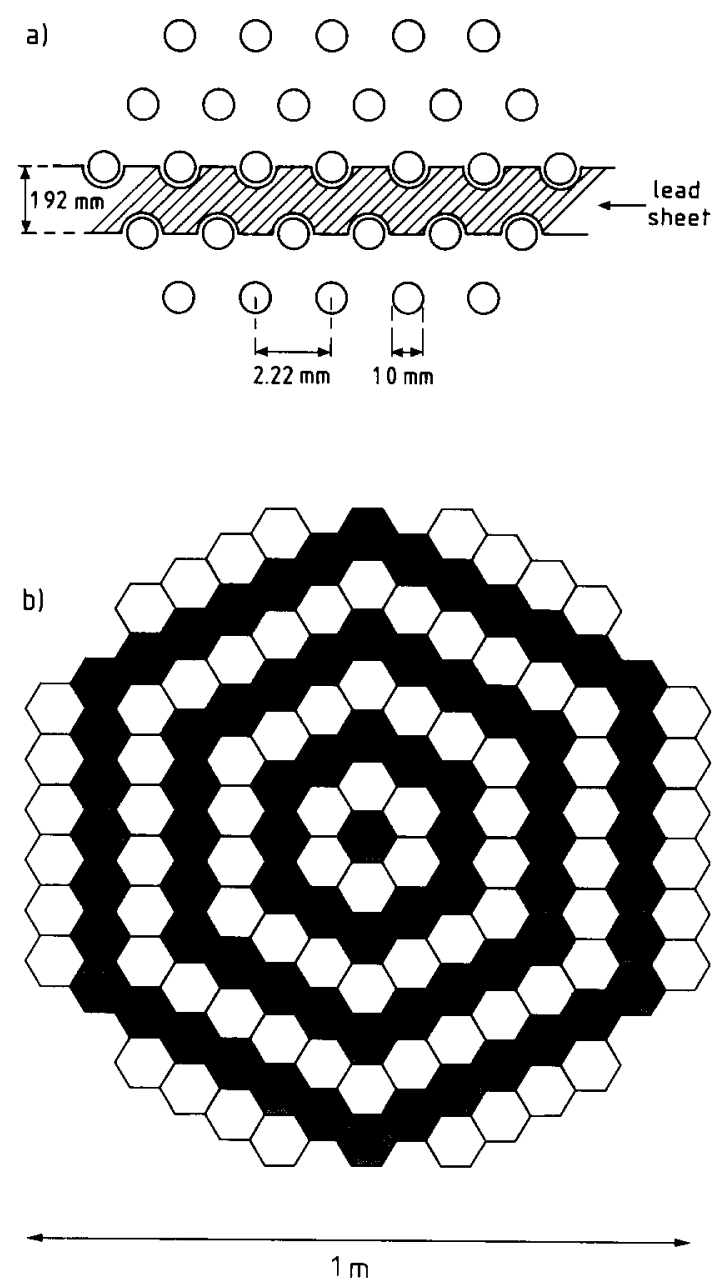

Fig. 1. Detail of the front face of the calorimeter (a) and the lateral structure of the detector as a whole (b).

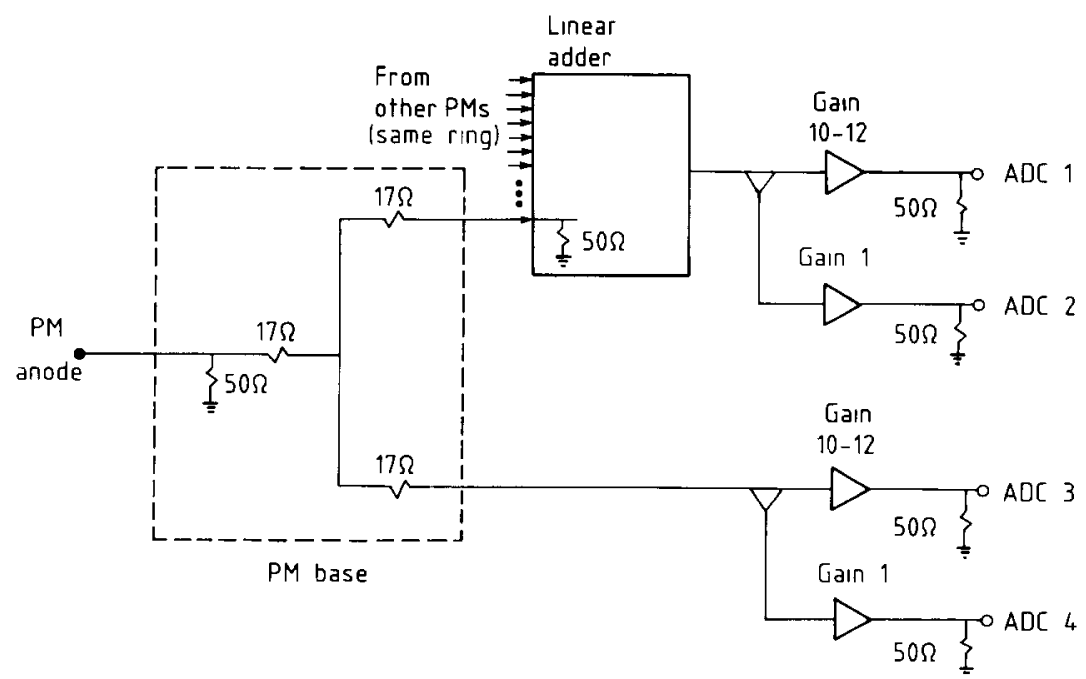

Fig. 2. The handling of the photomultiplier signals. See text for details. 
amounts to $7.2 \mathrm{~mm}$, the effective Molière radius $\left(R_{\mathrm{M}}\right)$ $20 \mathrm{~mm}$, the effective nuclear interaction length $\left(\lambda_{1}\right) 21$ $\mathrm{cm}$ [11] and the average density $9.0 \mathrm{~g} / \mathrm{cm}^{3}$. The sampling fraction for showers is only $2.3 \%$. The detector is, therefore, $9.6 \lambda_{\mathrm{I}}$ deep and $4.7 \lambda_{\mathrm{I}}$ across.

The PM signals were handled as follows (see fig. 2). The anode signal was split into two equal parts by means of a passive splitter inside the base. One part went to the counting room where it was further fed into an active splitter, one output of which was sent unchanged into the ADC system (LeCroy 2280, equipped with a 12-bit charge ADC, type 2282E), the other output was amplified by a factor $\sim 10$ before being fed into a charge ADC of the same type. The other half of the anode signal went into a linear adder, where it was combined with tower signals from the same hexagonal ring. The resulting ring sum signals were treated in the same way as the signals that were directly sent to counting room.

The gain in the PM tube was set to deliver $\sim 4$ $\mathrm{pC} / \mathrm{GeV}$ to both parts of the split signal in the central detector region, gradually going up to $\sim 20 \mathrm{pC} / \mathrm{GeV}$ in the outer rings, for the unamplified channels. The ADC gain was 4 counts $/ \mathrm{pC}$. This procedure was chosen to reduce the effects of the insufficient dynamic range of our PM/ADC system and to be sensitive to small energy deposits far away from the shower axis.

For the data discussed in this article, the ADC gate width was 400 ns. Sparse data readout was enabled: signals smaller than 4 counts above the pedestal value were not recorded. This corresponds to a cutoff of 5 $\mathrm{MeV}$ energy deposits in the amplified channels of the outer rings, gradually going up to $25 \mathrm{MeV}$ in the central-detector region. In this way, we could detect energy deposits ranging from $\sim 200 \mathrm{GeV}$ (the full range of the $\mathrm{ADC}$ for the unamplified channels in the central region) down to $5 \mathrm{MeV}$ (the cutoff limit in the amplified channels in the outer rings), which corresponds to a dynamic range of more than four orders of magnitude. Nevertheless, as will be shown in section 5 , this turned out to be insufficient. In particular, the hadronic signals at low energy were systematically underestimated by a significant amount.

\subsection{The beam line}

The measurements were performed in the $\mathrm{H} 2$ beam line of the SPS at CERN. The detector was mounted on a platform that could move horizontally and vertically with respect to the beam line, with an accuracy of about $1 \mathrm{~mm}$. Moreover, the detector could be rotated around its vertical axis, so that the particles could be sent into the detector at a chosen angle $\theta_{z}$ (usually a few degrees) to the fiber axis. The accuracy of the angular movement was better than $0.1 \mathrm{mrad}$. During the beam tests we found that the detector was slightly tilted vertically with respect to the beam line, by a few tenths of a degree.

Behind the detector, a smaller (20-tower) prototype of the same structure was installed. In practice, we used this detector to identify and remove muons from the data samples [12]. About $12 \mathrm{~cm}$ upstream from the SPACAL calorimeter, a preshower detector (PSD) was mounted. This preshower detector consisted of an absorber $1.14 X_{0}$ tungsten $+0.53 X_{0}$ lead), followed by a scintillation counter (S6). The signal in this scintillator provided separation between electron and pion events [8]. Further upstream from the calorimeters, a trigger counter telescope was installed. It consisted of five scintillation counters (S1-S5) and two drift chambers with $x, y$ readout $(\mathrm{BC} 1, \mathrm{BC} 2)$. The layout is shown in fig. 3 .

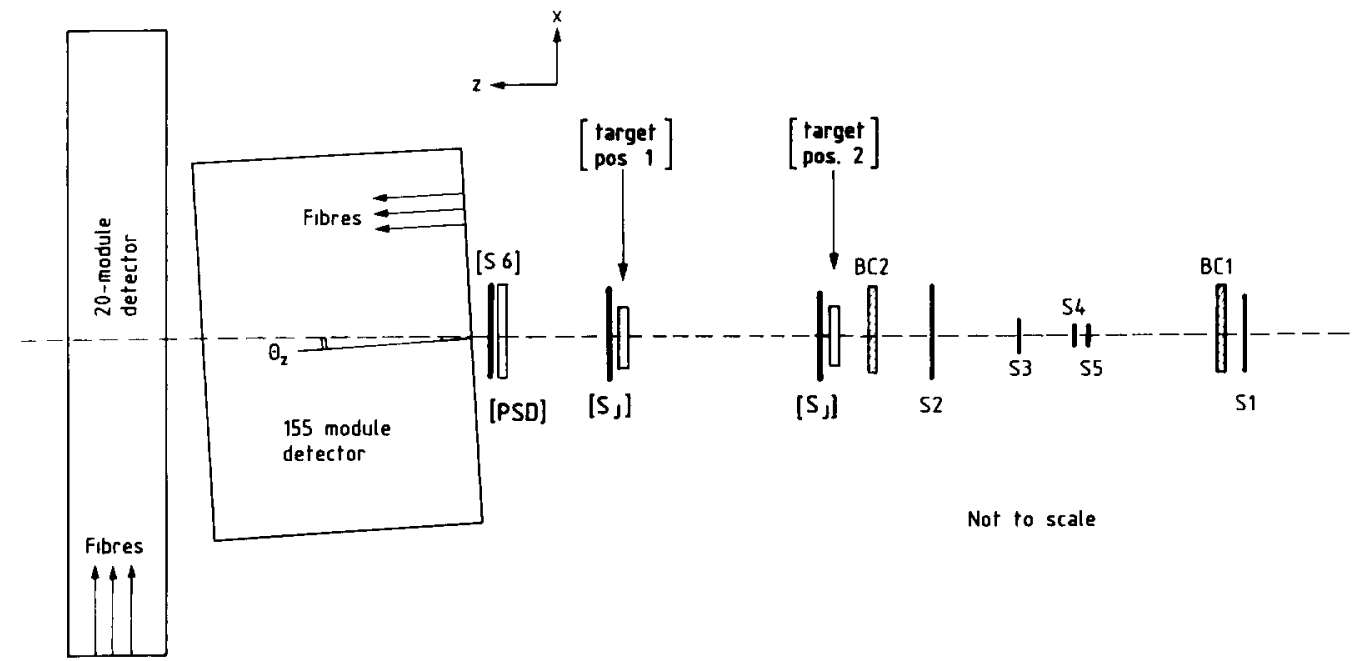

Fig. 3. Layout of the beam line, as seen from above. Elements that were not always in place during the beam tests are indicated in brackets. In our convention, the angle $\theta_{z}$ shown in the figure has a positive sign 
Beams of electrons and negative pions at 5.0, 9.7, $20,40,80$ and $150 \mathrm{GeV}$ were sent into the detector at an angle $\theta_{z}$ with the fiber axis. For most of the results reported here, $\theta_{2}$ was $3^{\circ}$. The beam particle rates were $10^{2}-10^{3}$ events per $2.6 \mathrm{~s}$ spill. The spills were repeated every $14 \mathrm{~s}$. At high energies ( $\geq 40 \mathrm{GeV})$ the beam were very clean, the contamination of electrons (pions) in the pion (electron) beam being far below the $1 \%$ level. At lower energies this was different. The event samples were cleaned up using data from the preshower detector. At 5 and $9.7 \mathrm{GeV}$, where the beams were most contaminated, the PSD signal was part of the trigger. For electrons, the signal was required to be above a threshold, set at $\sim 7$ times the minimum ionizing value, for pions the signal was required to be below this threshold.

The pion beams also contained some fraction of muons, varying from $\sim 2 \%$ at $150 \mathrm{GeV}$ to $\sim 80 \%$ at 5 $\mathrm{GeV}$. The elimination of these muons from the pion event samples will be described in detail in section 3 .

As an attempt to simulate jet detection with the calorimeter, we measured the reaction products of pions interacting in a target placed upstream of the detector. The target, a $10 \mathrm{~cm}$ thick block of paraffin, was installed at either $44 \mathrm{~cm}$ or $147 \mathrm{~cm}$ upstream of the calorimeter front face. We will refer to these configurations as position 1 and 2, respectively. Interactions in this target were selected with a scintillator counter $\left(\mathrm{S}_{\mathrm{J}}\right)$ downstream of the target (see fig. 3). For these measurements, the $S_{\mathrm{J}}$ signal was required to be at least twice the minimum ionizing (mip) value and was part of the trigger. Interaction trigger measurements were done with 40,80 and $150 \mathrm{GeV}$ pions. Since the preshower detector (S6, see fig. 3) covered only an area of $15 \times 15 \mathrm{~cm}^{2}$ in the central region of the calorimeter, it was removed during the interaction trigger measurements.

Off-line event selection required a single track, by cutting on the pulse height of the scintillation counters $\mathrm{S} 1, \mathrm{~S} 2$ and $\mathrm{S} 3$ between 0.5 and 1.7 times the mip value. The $x$ and $y$ coordinates measured in the two beam chambers had to agree within $1 \mathrm{~cm}$. Beam halo particles were removed by cuts on $x$ and $y$ in the beam chambers.

\section{Experimental data and analysis methods}

The SPACAL calorimeter was calibrated with 40 $\mathrm{GeV}$ electrons. About 1500 electrons were sent into the central region of each of the 155 individual towers, at an angle $\theta_{z}$ of $3^{\circ}$ to the fiber axis. In this way, the electromagnetic showers that developed were on average $\sim 95 \%$ contained in the tower concerned. Off-line, the calibration constants (the relation between pC's and GeV's for each individual tower) could be determined with a statistical precision of $\sim 0.3 \%$ from this data. However, systematic effects like fiber-to-fiber response fluctuations, high-voltage instabilities, etc. made the tower-to-tower calibration much less precisely known. We will come back to this point in sections 4.1 and 5.3.

The results described in the following sections were obtained by analyzing the following sets of data:

- Electrons and pions of 5.0, 9.7, 20, 40,80 and 150 $\mathrm{GeV}$ entering the SPACAL calorimeter at an angle $\theta_{z}=3^{\circ}$ with respect to the fiber axis. Typically, $\sim 3000$ electron and $\sim 5000$ pion events were accumulated per run, except at $5 \mathrm{GeV}$, where the event statistics were a factor two lower because of the low beam intensity.

- Pions of $40 \mathrm{GeV}$ entering the SPACAL detector at angles $\theta_{z}$ ranging from $0^{\circ}$ to $5^{\circ}$, in steps of $1^{\circ}$. Also here, $\sim 5000$ events were recorded at each angle.

- Electrons of $80 \mathrm{GeV}$ entering the detector at angles $\theta_{z}$ ranging from $0^{\circ}$ to $6^{\circ}$ and at $90^{\circ}$. These data were taken with a prototype of the SPACAL detector, consisting of 20 towers of the same structure.

- Matrix scans for $80 \mathrm{GeV}$ electrons and pions at $\theta_{z}=2^{\circ}$, entering the SPACAL detector at 49 different impact points, in contrast to the other mentioned data, where the pions always hit the SPACAL detector in the center. The data were taken in the form of a $7 \times 7$ grid scan, with the electrons covering an area of $12 \times 12$ $\mathrm{cm}^{2}$ in the central region of the calorimeter surface and the pions covering $24 \times 24 \mathrm{~cm}^{2}$. For each point, $\sim 2000$ events were recorded.

- Multiparticle runs where 40,80 or $150 \mathrm{GeV}$ pions interacted in a target placed either $44 \mathrm{~cm}$ or $147 \mathrm{~cm}$ upstream of the calorimeter. Reaction products of these interactions were detected with the SPACAL calorimeter. Per run, $\sim 10000$ events were accumulated.

For the analysis described in this article, clean and unbiased event samples for electrons and pions were needed. This was easiest to achieve for electrons, since the electron beams were very clean to start with, in particular also at low energies, where particle identification was most difficult. The few pions were removed with a cut on the signal in the preshower detector, requiring at least two mip for the event to be considered an electron. Muons were removed using the signal in the backing calorimeter. Any event with more than $125 \mathrm{MeV}$ deposited in this detector was discarded.

At high energies ( $\geq 40 \mathrm{GeV}$ ), establishing a clean and unbiased pion event sample was straightforward too. The beams were very clean and the few contaminating electrons and muons produced signals in the various detectors that were very different from the pion ones. Electron events were removed with a cut on the PSD signal, required to be smaller than two mip for the event to be considered a pion. The muon signals in SPACAL, well described by a Landau distri- 
bution with a most probable value of $4-5 \mathrm{GeV}$, could be efficiently removed by a cut on the total signal.

At lower energies, where the pion signals in SPACAL were correspondingly smaller while the signals from muons stayed essentially the same and the fraction of muons increased, the complete elimination of muons from the event samples gradually became more difficult. Also, the $\mathrm{e} / \pi$ separation by means of the PSD signal worked less well than at high energies.

Muons could be efficiently removed using the backing calorimeter signals. All muons traversing this detector produced a signal of at least $125 \mathrm{MeV}$. Of course, in this way one introduces in principle a bias in the pion samples, since these would be restricted to events producing less than $125 \mathrm{MeV}$ in the leakage detector. However, the effects of such a bias on the hadronic energy resolution and on the average hadronic signal were marginal, as will be shown in a forthcoming paper [12]. The fraction of pions showering in the $9.5 \lambda_{I}$ deep SPACAL detector and producing signals larger than $125 \mathrm{MeV}$ in the leakage calorimeter ranged from $<1 \%$ at $10 \mathrm{GeV}$ to $\sim 14 \%$ at $150 \mathrm{GeV}$. In this analysis, events with more than $125 \mathrm{MeV}$ deposited in the leakage calorimeter were discarded.

At the lowest energies $(5.0$ and $9.7 \mathrm{GeV})$, where the fraction of beam muons was very large (because of the very long distance between the detector and the beam production target), this cut did not completely remove all the contaminating events. Some muons may have missed the leakage detector because of multiple scattering in the big calorimeter. At $5 \mathrm{GeV}$, some of the muons were probably even completely stopped in the 2 $m$ of lead. And also some of the electrons deposited very little energy in the preshower detector and passed

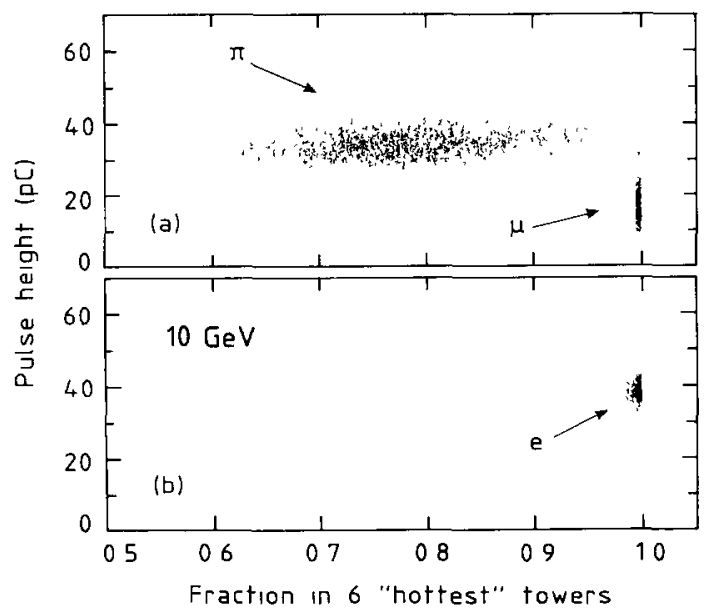

Fig. 4. The fraction $f_{6}$ of the total signal recorded in the 6 hottest SPACAL towers, versus the total SPACAL signal, for the events from the $9.7 \mathrm{GeV}$ pion beam (a). The same distribution for the $9.7 \mathrm{GeV}$ electron beam (b). the cut on the PSD signal. However, the remaining contamination could be easily recognized due to the fine lateral segmentation of the calorimeter. A criterion was developed, based on the energy deposit pattern in the calorimeter. The criterion exploits the fact that muons, which to first order only lose their energy through ionization, only produce signals in a very limited number of towers, contrary to showering pions. This is illustrated in fig. 4 , where the fraction $\left(f_{6}\right)$ of the total signal recorded in the six towers that gave the largest contributions to it is plotted against the total signal in the SPACAL calorimeter, for the particles from the $9.7 \mathrm{GeV}$ beam. From fig. $4 \mathrm{a}$, it can be seen that for example a cut $f_{6}<98 \%$ removes practically all the muons, while almost all pions pass such a cut. Also practically all electrons are removed by this cut (fig. 4b). In this way, it was possible to define very clean pion event samples with minimal bias, also at low energies. Even at $5 \mathrm{GeV}$, where electrons, pions and muons produced on average almost the same signal in the SPACAL calorimeter, the particles could be efficiently separated through cuts exploiting the differences in the energy deposit pattern.

In summary, electrons and pions were selected through the following cuts:

- S6 (PSD) $>2$ mip for electrons, S6 (PSD) $<2 \mathrm{mip}$ for pions,

- longitudinal leakage $<125 \mathrm{MeV}$ for all events, - $f_{6}<98 \%$ for pions, $f_{6}>98 \%$ for electrons.

The numbers of electron and pion events that were used for the present analysis are listed in the various tables.

\section{Electron detection}

\subsection{Energy resolution and signal uniformity}

The energy resolution for the detection of e.m. showers was studied as a function of the energy for electrons entering the calorimeter at an angle $\theta_{z}=3^{\circ}$. The preshower detector was in place for these measurements. A typical signal distribution, for $80 \mathrm{GeV}$ electrons, is shown in fig. 5. There are no significant deviations from a Gaussian line shape at the high-energy or the low-energy side of the peak. This was true at all energies for which results were obtained. Gaussian fits were performed without restrictions on the area around the peak value, all events were included. In table 1 the results of these fits are given.

The signal from fig. 5 was obtained by summing the signals of 37 calorimeter towers, the central tower plus the first three hexagonal rings surrounding this tower (see fig. 1b). Adding more towers did not significantly change the results. This is illustrated in fig. 6 , where a typical lateral e.m. shower profile is shown. The energy 


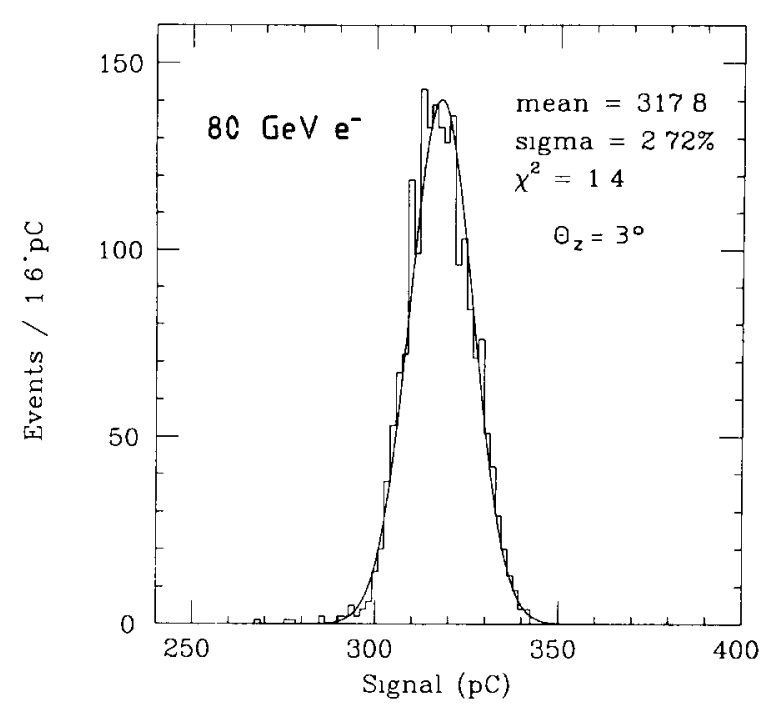

Fig. 5. Signal distribution for $80 \mathrm{GeV}$ electrons entering the Spaghetti Calorimeter at an angle $\theta_{z}=3^{\circ}$.

density falls very steeply, by three orders of magnitude over $10 \mathrm{~cm}$. The figure also shows that the noise level of this calorimeter is very low. Averaging over 1000 events, the lateral shower profile could be measured over six orders of magnitude. In fact, the results given in table 1 are not very different from the ones for the central tower alone. On average, $\sim 95 \%$ of the energy was deposited in this tower and the energy resolution improved only by $\sim 5 \%$ on average when the surrounding towers were taken into account as well. Since the e.m. showers are so narrow, the signal distributions are very sensitive to fiber-to-fiber response flıctuations [13] and, therefore, one may ask the question how representative these results, obtained in one point of the calorimeter, are for the detector as a whole. The answer is given in figs. 7 and 8 . Fig. 7 gives a distribution of the energy resolution for $40 \mathrm{GeV}$ electrons measured in each of the 155 individual towers of the detector. Each entry in this histogram represents the energy resolution measured at a different point (the center of a tower). The value obtained for the central tower and the statistical precision of the measurements

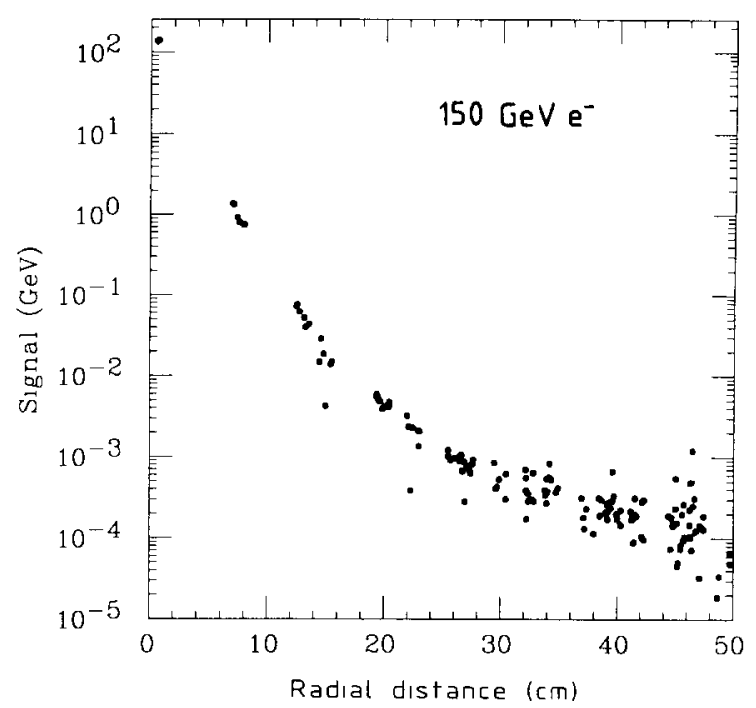

Fig. 6. Lateral shower profile for $150 \mathrm{GeV}$ electrons, averaged over 1000 events. The energy density ( $\mathrm{GeV}$ per tower) is shown as a function of the distance of the tower center to the center of gravity of the energy deposit profile.

entering this histogram are indicated in the figure. While fig. 7 concerns measurements in the centers of all the different SPACAL towers, fig. 8 shows the results of a fine scan covering an area of $144 \mathrm{~cm}^{2}$ in the central region of the detector. The scan was performed with $80 \mathrm{GeV}$ electrons, with the beam centered on 49 different impact points. The results are given as squares, the sizes of which indicate the numerical values (note that the chosen scale largely exaggerates the differences). In fig. 8 a the energy resolution is given, in fig. $8 \mathrm{~b}$ the average signal.

The spread in the distribution shown in fig. 7 is clearly larger than one would have expected on the basis of the statistical errors in the measurements. In fact, we found that the energy resolutions in the bottom half of the detector were on average significantly worse than the ones in the top half. This might indicate that the quality control of the fiber-to-fiber response fluctuations benefitted from a learning curve and gradually improved during the six months needed for the

Table 1

The e.m. energy resolution measured in the center of the detector at an angle $\theta_{z}=3^{\circ}$, with a $1.7 X_{0}$ preshower detector mounted $12 \mathrm{~cm}$ upstream of the calorimeter.

\begin{tabular}{rccccc}
\hline$E_{\mathrm{e}}[\mathrm{GeV}]$ & Events & $\langle$ signal $\rangle[\mathrm{pC}]$ & $\sigma[\mathrm{pC}]$ & $\sigma / E[\%]$ & $\sigma / E(\mathrm{lead})[\%]$ \\
\hline 5.0 & 645 & $18.65 \pm 0.05$ & $1.33 \pm 0.04$ & $7.04 \pm 0.20$ & - \\
9.7 & 2492 & $36.73 \pm 0.04$ & $2.02 \pm 0.03$ & $5.49 \pm 0.08$ & - \\
20.0 & 1350 & $78.90 \pm 0.09$ & $3.23 \pm 0.06$ & $4.06 \pm 0.08$ & $3.66 \pm 0.39$ \\
40.0 & 2074 & $158.9 \pm 0.1$ & $5.24 \pm 0.08$ & $3.29 \pm 0.05$ & $2.73 \pm 0.19$ \\
80.0 & 1924 & $317.8 \pm 0.2$ & $8.58 \pm 0.14$ & $2.72 \pm 0.04$ & $2.07 \pm 0.15$ \\
150.0 & 1032 & $579.2 \pm 0.4$ & $12.74 \pm 0.28$ & $2.23 \pm 0.05$ & $1.48 \pm 0.15$ \\
\hline
\end{tabular}




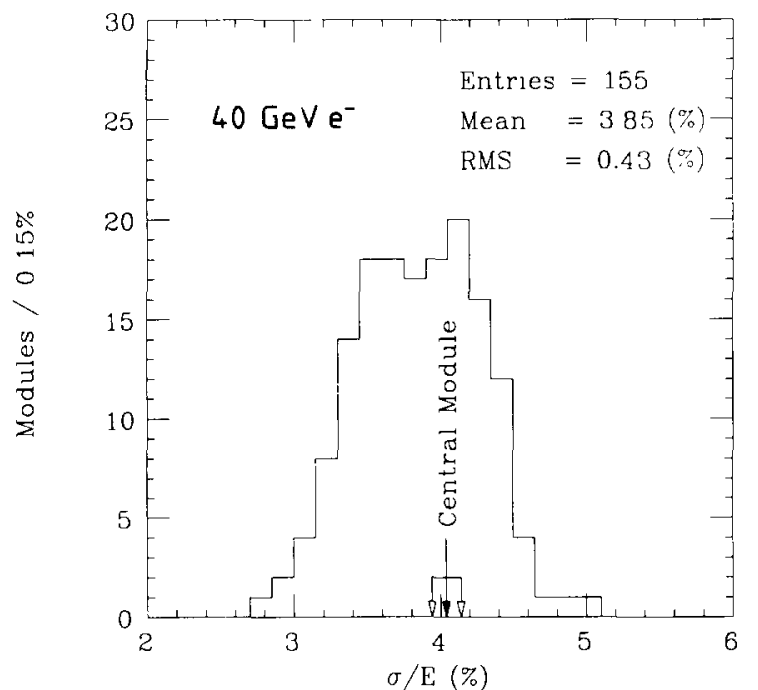

Fig. 7. Distribution of the energy resolutions for $40 \mathrm{GeV}$ electrons, measured in each of the 155 individual towers of the calorimeter. Each tower corresponds to one entry in this histogram. The value measured for the central tower is indicated, the statistical precision of the individual measurement results distributed in this histogram is shown as well.
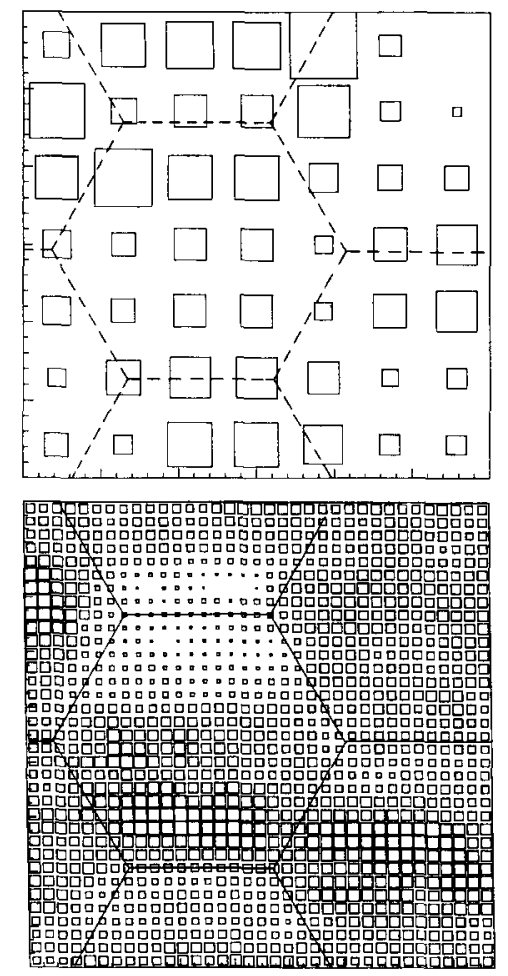

(b)

$$
=280 \mathrm{pC}
$$$$
\square=340 \mathrm{pC}
$$

Fig. 8 The energy resolution (a) and the average signal (b) for $80 \mathrm{GeV}$ electrons entering the central region of the calorimeter at a large number of impact points. The size of the squares indicates the numerical value (not proportional!). production of all the fibers. However, other explanations of this phenomenon cannot be ruled out. The average signal is even more sensitive to response fluctuations than the energy resolution. In the upper part of the central tower, the calorimeter response is systematically, up to $\sim 10 \%$, lower than the average value (fig. $8 \mathrm{~b}$ ). Measurements on the transmission of ambient light, which were done with a CCD camera after the beam tests were completed, revealed that the fibers in the top quarter of the central tower were significantly darker than the fibers in the rest of this tower. Similar measurements on six other, randomly chosen towers showed that the single-fiber response was much more uniform in these towers than in the central one. This is illustrated in fig. 9, which shows three of these towers and the central one. Fig. $8 \mathrm{~b}$ shows that, apart from the problem in the upper part of the central tower, the signal uniformity in the scanned detector area is quite good. In particular, there are no significant boundary effects between the various towers. This confirms earlier dedicated measurements intended to study such effects [9].

The data from fig. $8 \mathrm{~b}$ are presented as a histogram in fig. 10. The little bump at the low-energy side of the distribution represents the area with an anomalously low fiber response in the upper part of the central tower. Fig. 10 indicates how representative the calibration constants, obtained from measurements on electrons whose effective shower width is much smaller than the lateral tower size $\left(9.3 X_{0} \times 9.3 X_{0}\right)$ and which impact in one particular location, are for the tower as a whole. Therefore, the width of the distribution in fig. $10\left(\sigma_{\mathrm{RMS}} \sim 3 \%\right)$ may be interpreted as a measure for the tower-to-tower calibration accuracy in this region of the detector. The figure also shows that the electron response in the center of the detector is within $\sim 1 \%$ equal to the average response in this area. This means that systematic effects, due to signal non-uniformity, on e.g. the measured $\mathrm{e} / \pi$ signal ratio are very small.

Given the observed atypical imperfections in the central tower, we conclude that the energy resolutions measured in the center of the calorimeter and listed in table 1 are certainly not better than for the detector as a whole. The results from table 1 are graphically shown in fig. 11. They are well described with a fit of the following type:

$$
\begin{gathered}
\sigma / E=\frac{(12.9 \pm 0.3) \%}{\sqrt{E}}+(1.23 \pm 0.05) \% \\
\left(\chi^{2}=0.9 / \mathrm{df}\right)
\end{gathered}
$$

where $E$ is given in $\mathrm{GeV}$. One may also fit the data to an expression that adds the scaling and constant terms 
in quadrature. The result is:

$$
\begin{aligned}
\sigma^{2} / E^{2} & =\left(\frac{(15.7 \pm 0.2) \%}{\sqrt{E}}\right)^{2}+((1.99 \pm 0.05) \%)^{2} \\
\left(\chi^{2}\right. & =4.8 / \mathrm{df})
\end{aligned}
$$

Our data is not sufficiently sensitive to distinguish between these two expressions, one would need electrons at several hundred $\mathrm{GeV}$ for that purpose. Whether the scaling and constant terms have to be added in quadrature, linearly or in some intermediate way depends on the correlation between the processes responsible for these terms. The scaling term is determined by sampling fluctuations. The origin of the constant term is also well understood. Fig. 12 shows the calorimeter signal as a function of the $y$-coordinate of the impact point, determined with the beam chambers (see fig. 3). One sees an oscillating pattern of which the minima correspond to the situation where electrons enter the detector in the lead and the maxima to the situation in which the particles enter the detector in a plane composed of fibers and lead. The $1.9 \mathrm{~mm}$ period corresponds exactly to the vertical distance between two subsequent layers of fibers (see fig. 1a).

The lateral shower dimensions are very small, particularly in the early part of the shower. There, shower particles are predominantly emitted in the same direction as the incoming electron and the lateral shower dimensions, mainly determined by multiple scattering [2], are even small compared to the distance between two fibers. As a consequence, the sampling fraction for the early part of the shower and hence the total calorimeter signal depend on the impact point of the particles. Electrons entering the detector in a fiber will therefore on average produce larger signals than electrons entering in the lead. If one selects only elcctrons entering the detector in the lead (using the beam chamber information), the energy resolution clearly
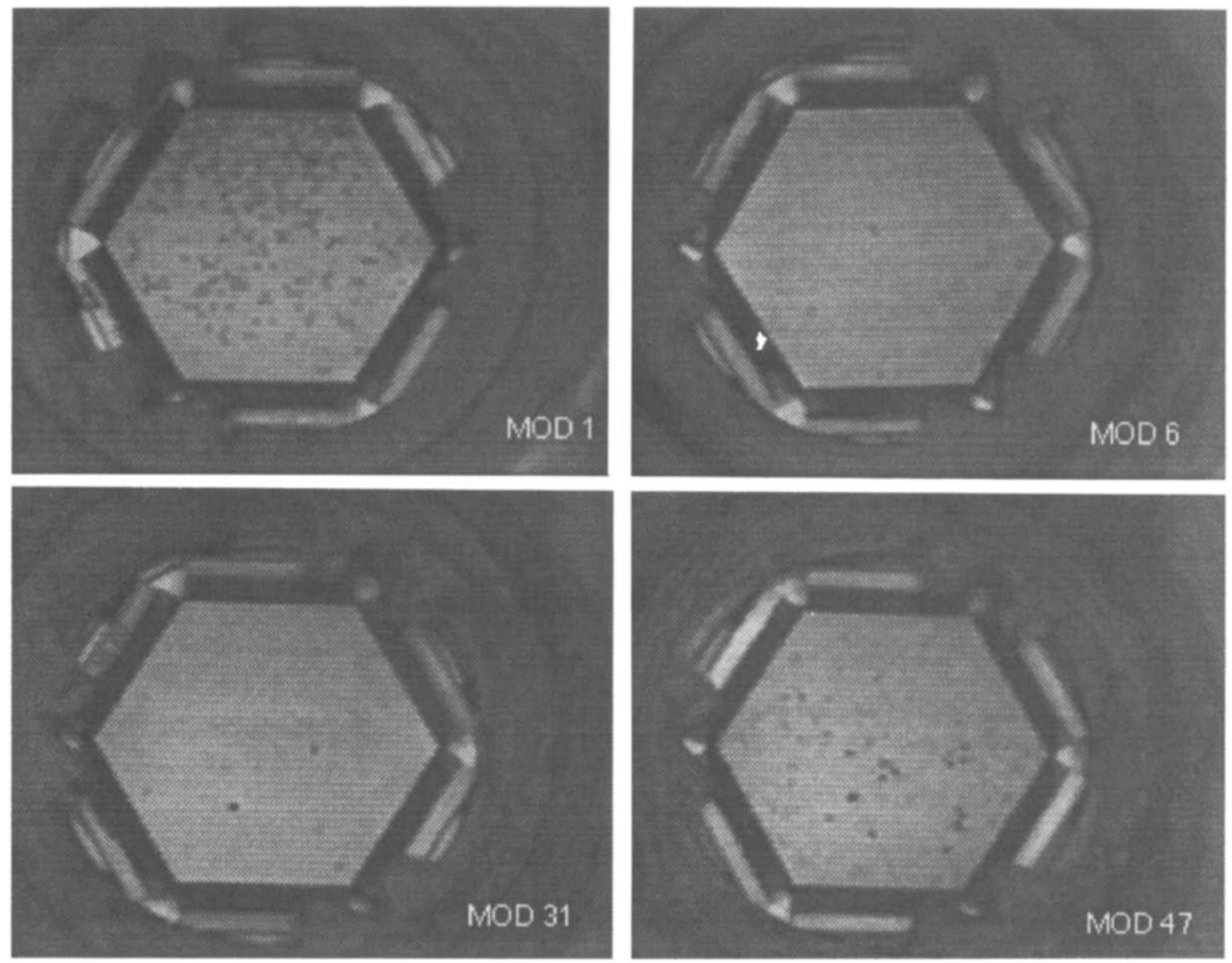

Fig. 9. Results of light transmıssion measurements with a CCD camera. Shown are the bunches of fibers from 4 individual towers. There are 1141 fibers in each bunch. The upper left picture shows the central tower of the detector, the others were chosen at random. 


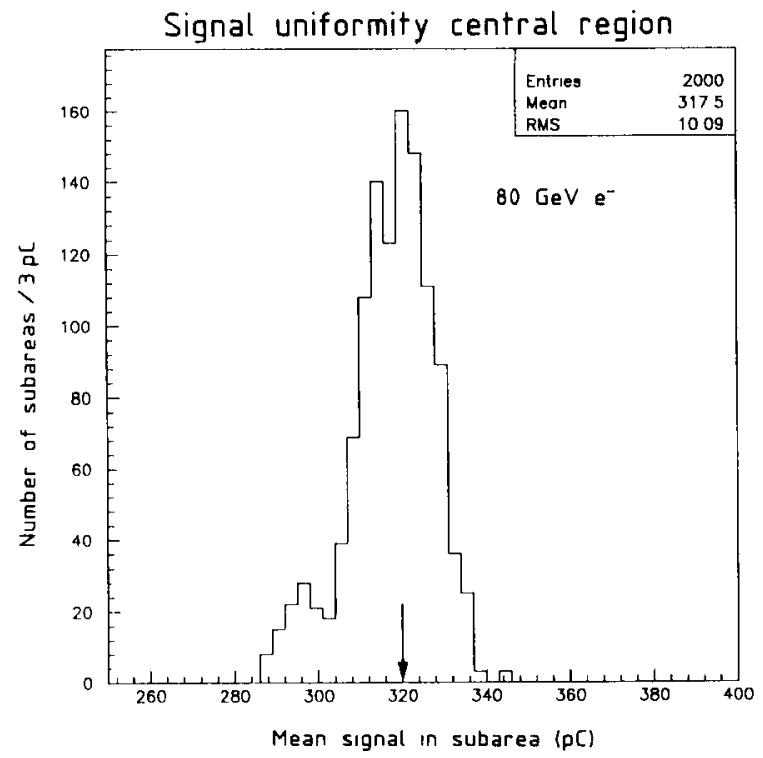

F1g. 10. Distribution of the calorimeter response to $40 \mathrm{GeV}$ electrons measured over an area of $144 \mathrm{~cm}^{2}$ in the form of a grid scan. Each entry in the histogram corresponds to the average response in a subarea of $\sim 3 \times 3 \mathrm{~mm}^{2}$. The electron response measured in the center of the calorimeter is indicated by an arrow.

improves, to the extent that the constant term is greatly reduced (see table 1 and fig. 11). At low energies, the beam dispersion made it increasingly impossible to determine the impact point of the electrons with sufficient (submillimetric) precision to make this selection possible. This dispersion is due to the preshower detector, which spreads the shower products more for lowenergy showers than for high-energy ones, thus washing out the effects of the fiber spacing on the energy

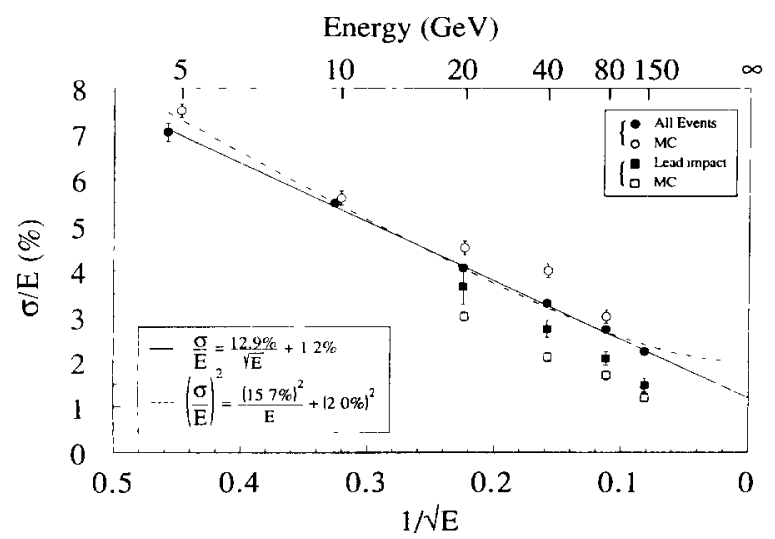

Fig. 11. The energy resolution for electrons as a function of energy, for $\theta_{z}=3^{\circ}$. The closed circles encompass all events, the closed squares are for electrons that enter the detector in the lead. Monte Carlo predictions are shown as open circles and squares.

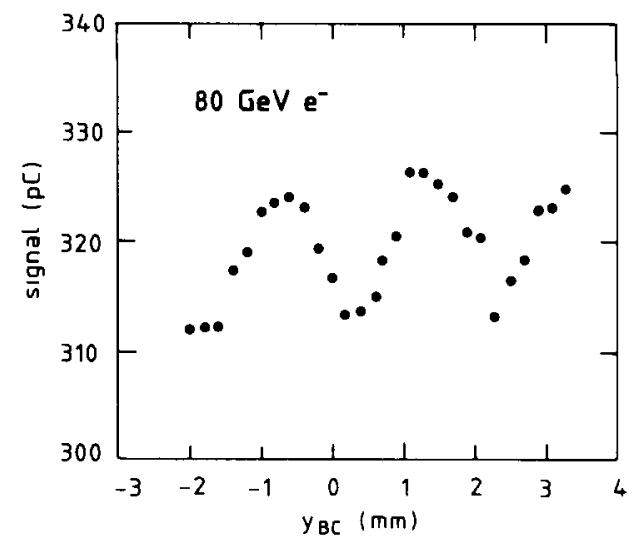

Fig. 12. The calorimeter signal as a function of the $y$-coordinate of the impact point. Data for $80 \mathrm{GeV} \mathrm{e}^{-}$.

resolution. Therefore, only results for $E \geq 20 \mathrm{GeV}$ are given.

We conclude that the process responsible for the deviations from a pure scaling behavior of the e.m. energy resolution is a) not completely uncorrelated to the sampling fluctuations, and b) energy dependent, since the fraction of the shower that is subject to this anomalous sampling in the early stage of the shower development is (weakly) dependent on the energy.

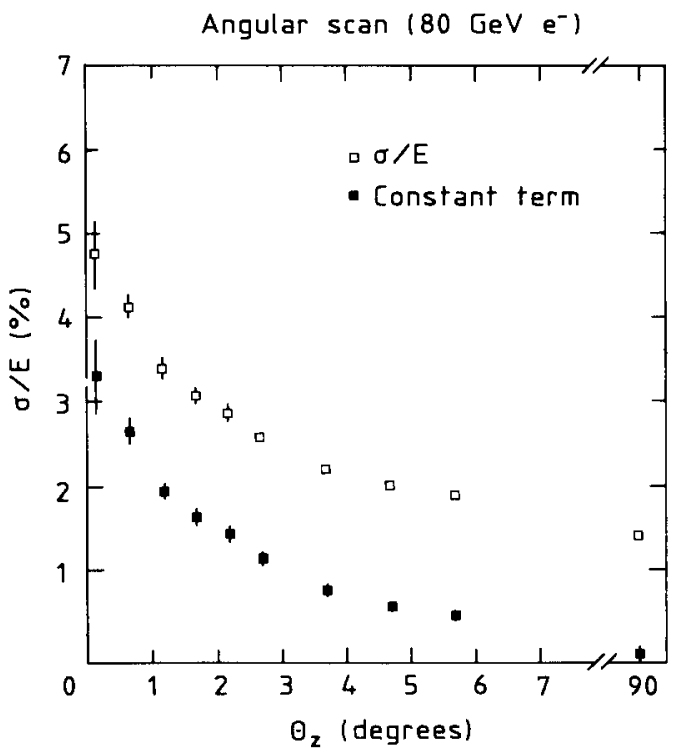

Fig. 13. The energy resolution $\sigma / E$ as a function of the angle $\theta_{z}$ between the particle direction and the fiber axis (open squares). The constant term $c$ in the e.m. energy resolution as a function of $\theta_{z}$, assuming that the results scale as $\sigma / E=$ $12.9 \% / \sqrt{E}+c\left(\theta_{2}\right)$, is shown as well (full squares). Results are for $80 \mathrm{GeV}$ electrons, measured in a 20 -tower prototype of the SPACAL detector. 
The latter point is in favour of eq. (1a), which includes an energy dependent deviation from $E^{-1 / 2}$ scaling. Also, the better $\chi^{2}$ of the fit suggest that the two terms should be added linearly. However, neither (1a) nor (1b) is theoretically completely justified and a correct formula is probably more complicated.

Fig. 11 also shows the results of Monte Carlo simulations (GEANT). These simulations did not include the effects of the preshower detector and the fiber cladding. The shower development was cut off at an energy of $10 \mathrm{keV}$. The results confirm the deviation from a pure scaling behavior, but tend to slightly overestimate the contribution of the constant term to the energy resolution. The Monte Carlo simulations also predict a considerable improvement in the energy resolution and a smaller sampling fraction (i.e. calorimeter signal) if only electrons entering in the lead are considered. The predicted improvement is somewhat larger than the one observed in practice (fig. 11). This difference is probably due to the finite position resolution of the beam chambers $\mathrm{BC} 1$ and $\mathrm{BC} 2$ (see fig. 3) and to the PSD effect mentioned above, which did not allow to achieve perfect separation between electrons entering the calorimeter in lead or in a fiber.

The origin of the constant term being understood, it is no surprise that it depends very strongly on the angle $\theta_{z}$. Fig. 13 shows the energy resolution for $80 \mathrm{GeV}$ electrons as a function of $\theta_{z}$. Earlier, more systematic studies with small prototype modules showed that if the data were fitted to an expression $\sigma / E=a / \sqrt{E}+c$, the scaling term parameter $(a)$ was almost independent of $\theta_{z}$, whereas $c$ depended strongly on this angle [9]. Assuming that $a$ is independent of $\theta_{z}$, we can derive $c\left(\theta_{z}\right)$ from the measured energy resolution at $80 \mathrm{GeV}$. The results, also given in fig. 13, show that the constant term has almost completely vanished for $\theta_{z}=90^{\circ}$, and increases strongly below $\theta_{z}=5^{\circ}$.

The variations in the energy of the incoming electrons, which in principle also contribute to the energy resolution, were neglected in the analysis. During our measurements, the $\Delta p / p$ of the electrons ranged from $0.7 \%$ at $5 \mathrm{GeV}$ to $0.1 \%$ at $150 \mathrm{GeV}$.

\subsection{Effects of the preshower detector}

The results discussed in the previous subsection were obtained with the $1.7 X_{0}$ thick preshower detector installed $12 \mathrm{~cm}$ upstream of the calorimeter. In principle, this preshower detector will have deteriorated the energy resolution of the calorimeter, since it absorbed some fraction of the shower energy, with relatively large event-to-event fluctuations. In this subsection, we will investigate to what extent the calorimeter's energy resolution has been affected. Also, the preshower detector absorbed a larger fraction of the energy of electrons than for the pions. In order to be able to determine the $e / h$ value correctly, this effect has to be evaluated as well.

The preshower detector was calibrated with the signals from beam muons, which were assumed to be minimum ionizing particles for this purpose. From the composition of the absorber, one can calculate the most probable value for the energy loss of mip's in this preshower detector: $13.7 \mathrm{MeV}$. This sets the energy scale for the observed electron signals. However, it should be realized that e.m. showers are less efficiently sampled than muons in high- $Z$ calorimeters. Typically, an $\mathrm{e} / \mathrm{mip}$ value of $\sim 0.6$ is found $[3,14]$. This result applies to showers that are completely absorbed. The early part is more efficiently sampled, with $\mathrm{e} / \mathrm{mip} \sim 0.8$ for the first few radiation lengths [3]. So in practice we used an energy scale of $13.7 / 0.8=17.1 \mathrm{MeV} / \mathrm{mip}$ for the electron signals. The average shower energy deposited in the preshower detector is listed in table 2. The fractional energy loss is given as a function of the electron energy in fig. 14. The step at low energy is caused by the trigger threshold applied for the measurements at 5 and $9.7 \mathrm{GeV}$ (see section 2.2). Results of Monte Carlo simulations (GEANT) in which the

Table 2

The average energy deposited in the $1.7 X_{0}$ preshower detector, and the effect of this detector on the energy resolution measured with the calorimeter. Column 4 lists the measured energy resolutions ignoring the PSD signals. In column 5, the resolutions obtained by adding the PSD and SPACAL signals event-by-event are given.

\begin{tabular}{rllll}
\hline $\begin{array}{l}E_{\mathrm{e}} \\
{[\mathrm{GeV}]}\end{array}$ & $\begin{array}{l}\Delta E \text { PSD } \\
{[\mathrm{GeV}]}\end{array}$ & $\begin{array}{l}\Delta E / E \\
{[\%]}\end{array}$ & $\begin{array}{l}\sigma / E \\
\text { no PSD } \\
{[\%]}\end{array}$ & $\begin{array}{l}\sigma / E \\
\text { with PSD } \\
{[\%]}\end{array}$ \\
\hline 5.0 & & & $7.04 \pm 0.20$ & $6.92 \pm 0.20$ \\
9.7 & $0.236 \pm 0.024$ & $4.72 \pm 0.47$ & $5.49 \pm 0.08$ & $5.29 \pm 0.07$ \\
20.0 & $0.298 \pm 0.030$ & $3.07 \pm 0.31$ & $4.06 \pm 0.08$ & $3.99 \pm 0.08$ \\
40.0 & $0.166 \pm 0.017$ & $0.83 \pm 0.08$ & $3.29 \pm 0.05$ & $3.24 \pm 0.05$ \\
80.0 & $0.221 \pm 0.022$ & $0.55 \pm 0.06$ & $2.72 \pm 0.04$ & $2.71 \pm 0.04$ \\
150.0 & $0.274 \pm 0.027$ & $0.34 \pm 0.03$ & $2.23 \pm 0.05$ & $2.28 \pm 0.05$ \\
\hline
\end{tabular}


energy deposit in the preshower detector was calculated, are also shown in this figure. They agree within $\sim 10 \%$ with the experimental values, except of course at 5 and $9.7 \mathrm{GeV}$, where the trigger threshold $\left(E_{\mathrm{PSD}}>7\right.$ mip) was applied. Mainly because of the trigger threshold that was applied for the 5 and $9.7 \mathrm{GeV}$ data taking, the effect of the preshower detector on the $e / \pi$ signal ratio is not negligible at these low energies. Energy loss in the preshower detector is also partly responsible for the signal alinearity observed at low energies (see section 5.3).

The effect of the preshower detector on the energy resolution for electrons was determined by adding the signals from this detector event-by-event to the calorimeter ones. The results, listed in table 2, show that the improvement in the energy resolution was barely significant. This is no surprise, since the preshower detector absorbed at maximum only a few percent of the shower energy, and only the fluctuations in these few percent might affect the energy resolution. On the other hand, the linearity of response clearly improved at low energy when the energy loss in the PSD was taken into account (see section 5.3). For completeness, it should be mentioned that the fits describing the measured energy resolutions (eqs. (1a) and (1b)) took the energy loss in the PSD into account, i.e. the $5 \mathrm{GeV}$ point was taken at $4.76 \mathrm{GeV}$, etc. The effect of this on the results of the fits was smaller than the statistical errors in the coefficients.

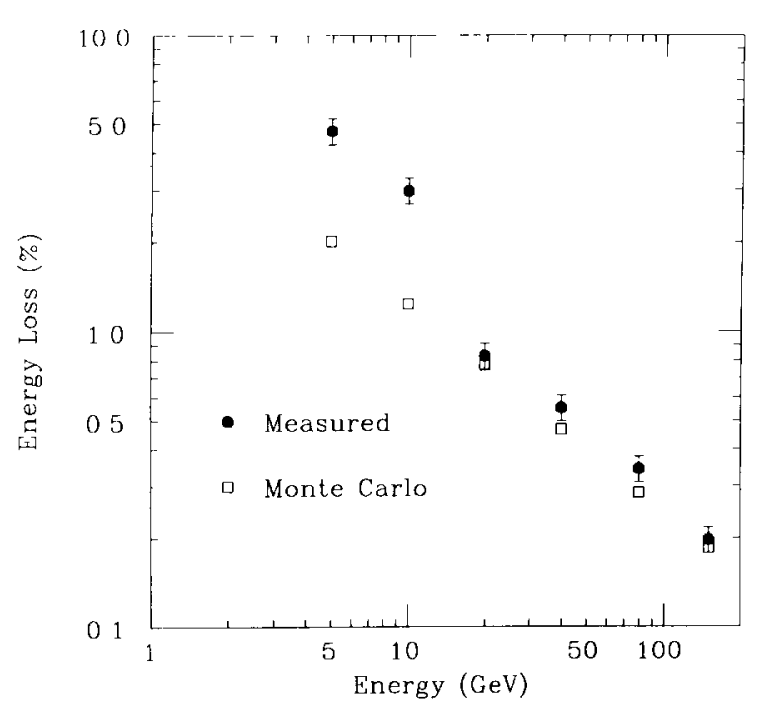

Fig. 14. The fractional energy loss in the $1.7 X_{0}$ thick preshower detector as a function of the electron energy. Results of $\mathrm{MC}$ sımulations (GEANT) are given as well.

\section{Single-pion detection}

\subsection{Energy resolution}

The energy resolution for the detection of hadronic showers was studied as a function of the energy for negative pions entering the calorimeter at an angle $\theta_{z}=3^{\circ}$. Pure samples of pion events were obtained as described in section 3 . The pion signals were obtained by adding the signals from all calorimeter towers.

Typical signal distributions, for $9.7,40$ and $150 \mathrm{GeV}$ $\pi^{-}$, are shown in figs. $15 \mathrm{a}, 15 \mathrm{c}$ and $15 \mathrm{e}$. The shape of these distributions is not very well described by a Gaussian curve, especially at $150 \mathrm{GeV}$, where a clear high-energy tail is observed. The reason for this is well understood. Hadronic showers exhibit large fluctuations in their longitudinal energy deposit profile. Because of light attenuation in the fibers, the calorimeter response depends on the depth at which the light is produced. This effect is responsible for the observed asymmetric signal distributions.

The light attenuation curve of the fibers was measured in the following way. A beam of $40 \mathrm{GeV}$ electrons was steered into a Spaghetti Calorimeter which was oriented at $\theta_{z}=90^{\circ}$, i.e. with the fibers running perpendicular to the beam line. These measurements were performed with the 20 -tower prototype calorimeter. The signal of the e.m. showers was measured as a function of the distance $z$ between the impact point and the PM tubes. The results are given in fig. 16. The experimental points can be described by a sum of exponentials:

$$
\begin{aligned}
I(z)[\mathrm{pC}]= & 102\left[\mathrm{e}^{-z / 110}+0.85 \mathrm{e}^{(z-44) / 110}\right] \\
& +124 \mathrm{e}^{-z / 077}
\end{aligned}
$$

(with $z$ in meters), which accounts for two exponential attenuation processes and the effect of light reflection at the aluminized open end (reflection coefficient 0.85 [9]) in the $2.2 \mathrm{~m}$ long fibers. The curve in fig. 16 corresponds to this expression. Far away from the PMs, where on average most of the light is produced by a shower, the effective attenuation length measured over the first meter amounts to $7-8 \mathrm{~m}$, but in the second half of the detector, the detector response rises fairly sharply. The short component in the light attenuation curve of scintillating fibers, $77 \mathrm{~cm}$ in our case, is due to light travelling (partly) through the fiber cladding [15]. Reflection losses at the cladding-air interface are causing the much stronger attenuation for this light component. It is clear that especially the response to high energy hadrons, which may occasionally deposit quite a large fraction of their shower energy beyond $5 \lambda_{1}$, may be affected by light attenuation. This is illustrated in fig. 17, where the calorimeter signal is shown versus the longitudinal center of gravity of the light 
production. This effective shower depth was determined from the displacement of the lateral center of gravity with respect to the impact point of the particles (a consequence of the angle $\theta_{z}$ ) [10], exploiting the good position resolution of the detector. The effect of light attenuation is clearly visible, especially at 150 $\mathrm{GeV}$, in that the average pion signal rises at increasing depth. In fact, figs. $17 \mathrm{~b}$ and 16 are equivalent, with the difference that $\theta_{z}$ was $3^{\circ}$ instead of $90^{\circ}$.

As a consequence of light attenuation, the measured energy resolution is clearly worse than indicated by the widths of the bands in fig. 17. This width is determined by the fluctuations in the shower development itself. Moreover, the resulting signal distribution is non-Gaussian as a consequence of the non-Gaussian event distribution in depth. All these effects are clearer at higher energy, because of the following reasons: a) The energy resolution due to sources other than light attenuation is much smaller at high energy. b) The effective depth of the light production is better determined at high energy because of the better lateral position resolution. This is illustrated by the fact that
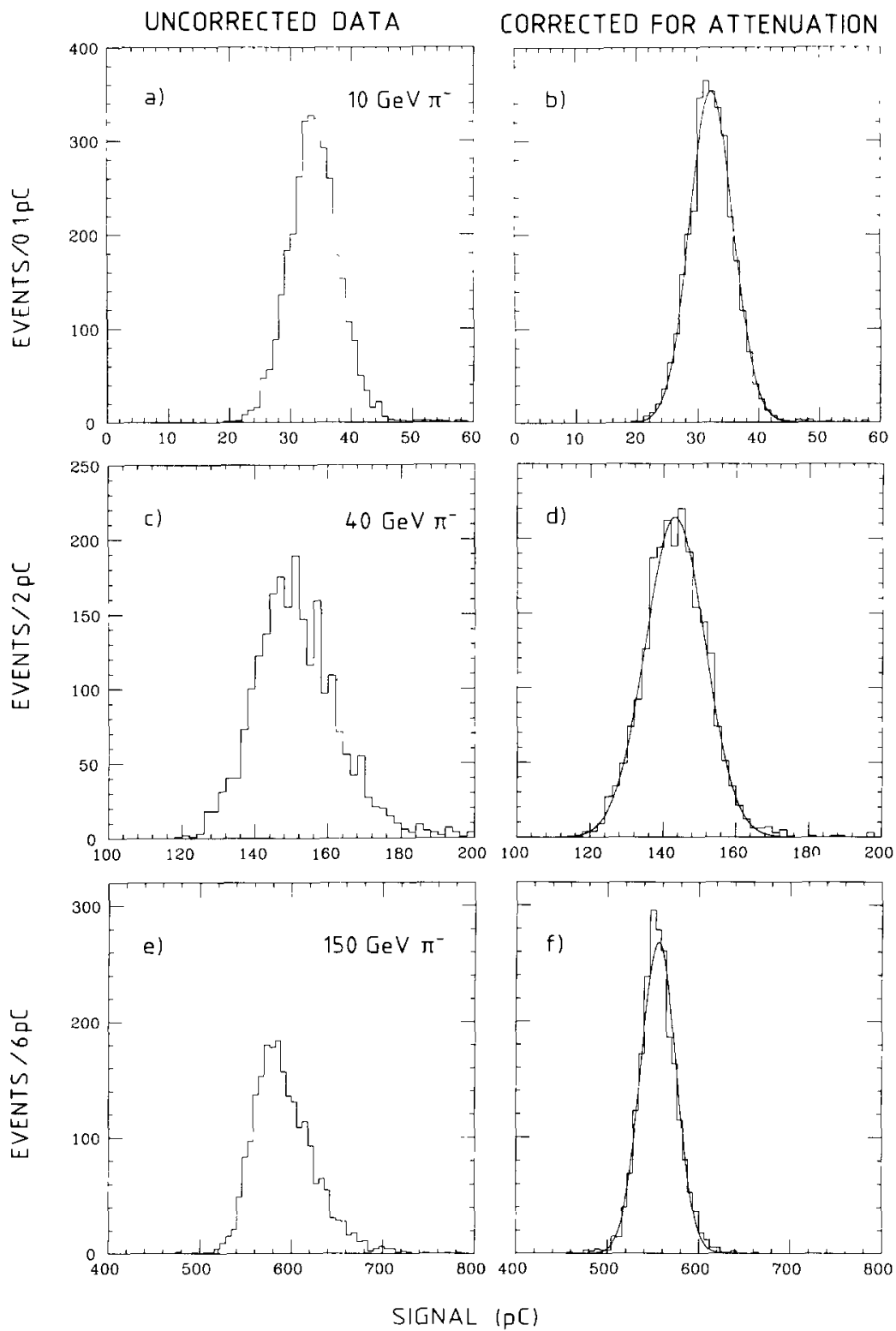

Fig. 15. Signal distributions for $9.7,40$ and $150 \mathrm{GeV}$ pions entering the Spaghetti Calorimeter at an angle $\theta_{z}=3^{\circ}$. The measured distributions are shown on the left $(\mathrm{a}, \mathrm{c}, \mathrm{e})$. The distributions on the right hand side were obtained after correcting for the effects of light attenuation in the fibers (b, d, f). The curves are the results of Gaussian fits. 


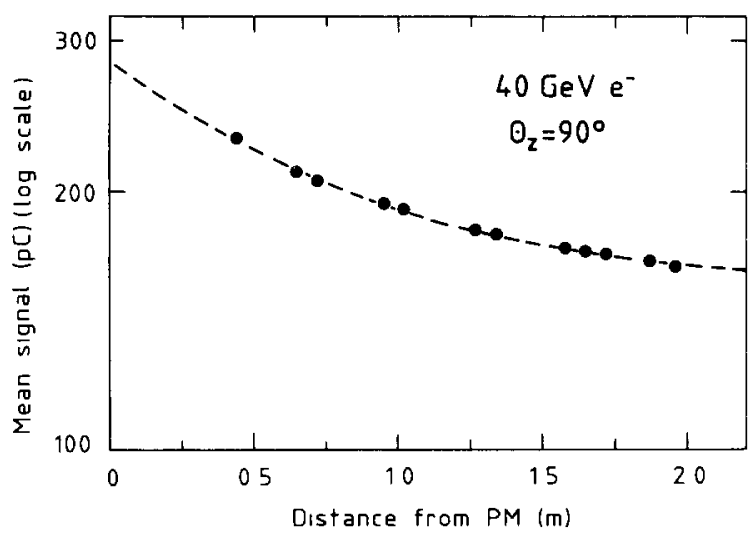

Fig. 16. The average calorimeter signal for $40 \mathrm{GeV}$ electrons entering the detector perpendicularly $\left(\theta_{z}=90^{\circ}\right)$, as a function of the distance $z$ between the impact point and the PM tubes.

at $9.7 \mathrm{GeV}$ quite a few points at unphysical values are found $\left(x_{\mathrm{CAL}}-x_{\mathrm{BC}}>0\right)$. c) The longitudinal dimensions of high energy showers are larger, so that more light is produced in the region where relatively strong light attenuation occurs.

If one knows the effective depth $(z)$ of the light production event-by-event, one can of course eliminate the effects of light attenuation by weighting the signals with a factor $I(2.2) / I(z)$ (see eq. (2)). The results of such a procedure are shown in figs. $15 \mathrm{~b}, 15 \mathrm{~d}$ and $15 \mathrm{f}$. As may be expected, the relative improvement is much larger at high energy. As a result of this weighting procedure, the energy resolution at $150 \mathrm{GeV}$ improves considerably and the signal distribution is much better described by a Gaussian.

The results on the energy resolution for single pions are summarized in table 3 , which lists for each energy the number of events, the average calorimeter signal and the energy resolutions $\sigma / E$ before and after correcting for the effects of light attenuation. Gaussian fits were performed without restrictions on the area around the peak value. The energy resolutions before and after correcting the effects of light attenuation are also shown as a function of the pion energy in fig. 18 . When fitting the energy resolutions to an expression of

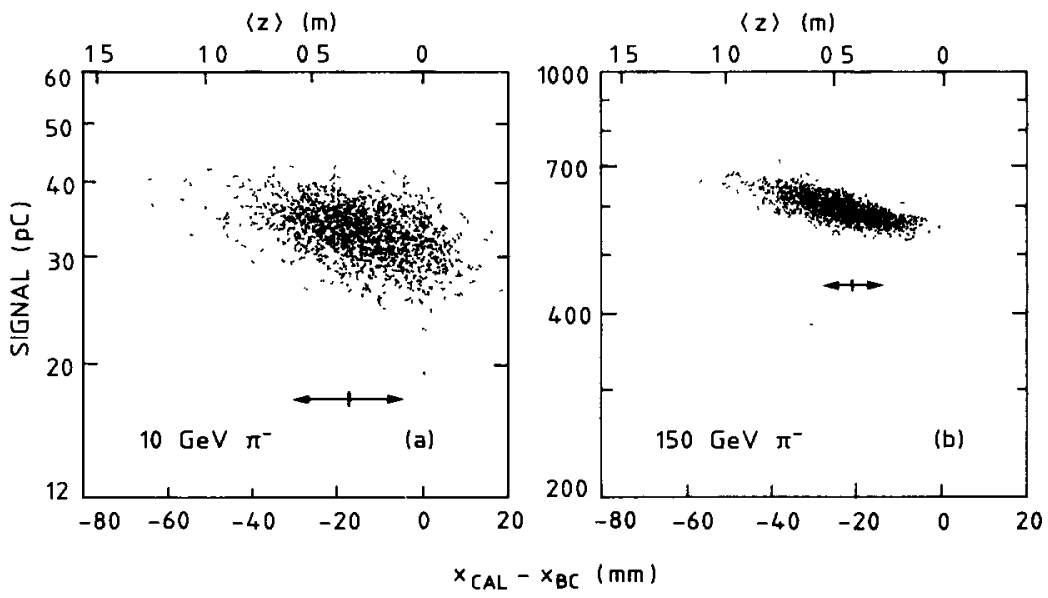

Fig. 17. Scatter plots showing the calorimeter signal versus the longitudinal center of gravity of the light production (top horizontal axis). This depth was determined from the lateral displacement of the center of gravity of the shower with respect to the particle's impact point (bottom horizontal axis). The arrows indicate the precision of the position measurements. Data for $9.7 \mathrm{GeV} \pi^{-}$(a) and $150 \mathrm{GeV} \pi^{-}$(b), entering the detector at $\theta_{z}=3^{\circ}$.

Table 3

The energy resolution for single pions, before (column 4) and after correcting for the effects of light attenuation in the fibers (column 5) and of residual non-compensation (column 6).

\begin{tabular}{rccccc}
\hline$E_{\pi}[\mathrm{GeV}]$ & Events & $\langle$ signal $\rangle[\mathrm{pC}]$ & $\sigma / E[\%] / \chi^{2}$ & $\sigma / E\left(\lambda_{\text {att }}=\infty\right)[\%] / \chi^{2}$ & $\sigma / E\left(R_{\mathrm{p}} \operatorname{corr}\right)[\%] / \chi^{2}$ \\
\hline 5.0 & 248 & $16.99 \pm 0.20$ & $15.6 \pm 0.7 / 0.55$ & $14.8 \pm 1.5 / 1.2$ & - \\
9.7 & 3213 & $34.09 \pm 0.07$ & $11.73 \pm 0.15 / 1.1$ & $11.05 \pm 0.14 / 1.0$ & - \\
20.0 & 1893 & $73.25 \pm 0.14$ & $8.25 \pm 0.13 / 1.4$ & $7.50 \pm 0.12 / 0.8$ & - \\
40.0 & 2286 & $151.6 \pm 0.2$ & $6.96 \pm 0.10 / 2.0$ & $5.75 \pm 0.09 / 1.2$ & - \\
80.0 & 2479 & $309.2 \pm 0.4$ & $5.58 \pm 0.08 / 2.9$ & $4.40 \pm 0.06 / 1.8$ & $4.08 \pm 0.12 / 1.2$ \\
150.0 & 2208 & $593.4 \pm 0.6$ & $4.86 \pm 0.07 / 3.7$ & $3.46 \pm 0.05 / 1.9$ & $3.00 \pm 0.09 / 1.2$ \\
\hline
\end{tabular}



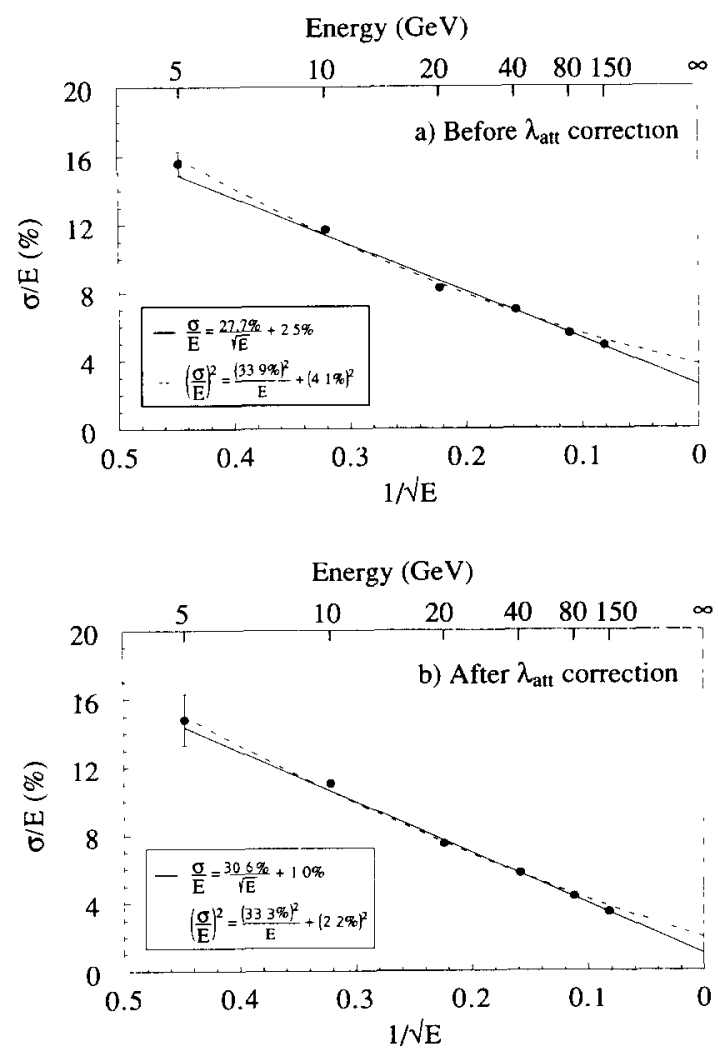

Fig. 18. The energy resolutions for single pions as a function of the pion energy, before (a) and after (b) correcting for the effects of light attenuation in the fibers. The curves are the results of least squares fits to the data.

the type $\sigma / E=a / \sqrt{E}+c$, we found the following results:

Before:

$$
\begin{aligned}
\sigma / E & =\frac{(27.7 \pm 0.6) \%}{\sqrt{E}}+(2.5 \pm 0.1) \% \\
\left(\chi^{2}\right. & =4.8 / \mathrm{df})
\end{aligned}
$$

after:

$\sigma / E=\frac{(30.6 \pm 0.5) \%}{\sqrt{E}}+(1.0 \pm 0.1) \%$

$$
\left(\chi^{2}=2.4 / \mathrm{df}\right)
$$

with $E$ given in $\mathrm{GeV}$. One may also fit the data to an expression that adds the scaling and constant terms in quadrature. This gives the following results:

Before:

$$
\begin{aligned}
\sigma^{2} / E^{2} & =\left(\frac{(33.9 \pm 0.4) \%}{\sqrt{E}}\right)^{2}+((4.1 \pm 0.1) \%)^{2} \\
\left(\chi^{2}\right. & =3.4 / \mathrm{df})
\end{aligned}
$$

after:

$$
\begin{aligned}
\sigma^{2} / E^{2} & =\left(\frac{(33.3 \pm 0.4) \%}{\sqrt{E}}\right)^{2}+((2.2 \pm 0.1) \%)^{2} \\
\left(x^{2}\right. & =2.0 / \mathrm{df}) .
\end{aligned}
$$

The curves in fig. 18 correspond to eqs. (3) and (4). As in the case of e.m. showers, one may argue whether the two terms have to be added linearly, in quadrature or otherwise. The fact that the effects causing a deviation from a pure scaling behavior are energy dependent works in favour of eq. (3). But, as for e.m. showers, neither of the two expressions is theoretically justified and a correct formula is definitely more complicated than the ones used here. Fig. 18 shows that only measurements at much higher energies than the ones used by us will allow to distinguish experimentally between the linear and the quadratic curves. It is interesting to note that the WA80 Collaboration, who measured the energy resolution of their uranium/ scintillator calorimeter for energies between 60 and $6400 \mathrm{GeV}$, obtained results that are favoring the linear expression [16].

The results given in eqs. (3) and (4) show that, although light attenuation in the fibers is a major contribution to the term that describes the deviation from a pure scaling behaviour, it does not explain this term completely. In an attempt to understand the origin of the remaining effects, we found that the width of the signal distributions could be further reduced if the calibration constants were deliberately changed with respect to their measured values. This is illustrated in fig. 19, which shows the energy resolution $\sigma / E$, normalized to its value for the nominal calibration constants, as a function of the calibration constant for the central tower. The width of the signal distribu-

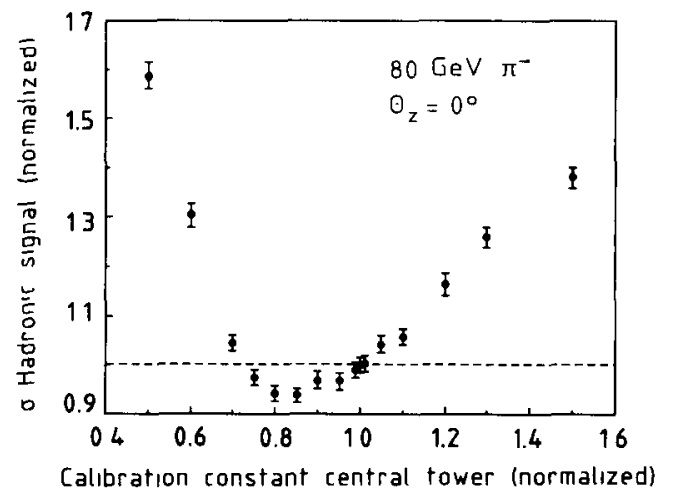

Fig. 19. The fractional width of the hadronic signal distribution as a function of the calibration constant for the central calorimeter tower. The scales are normalized to the values for the nominal calibration constant, determined from the calorimeter response to electrons hitting the center of this tower. Data for $80 \mathrm{GeV}^{-}$. 

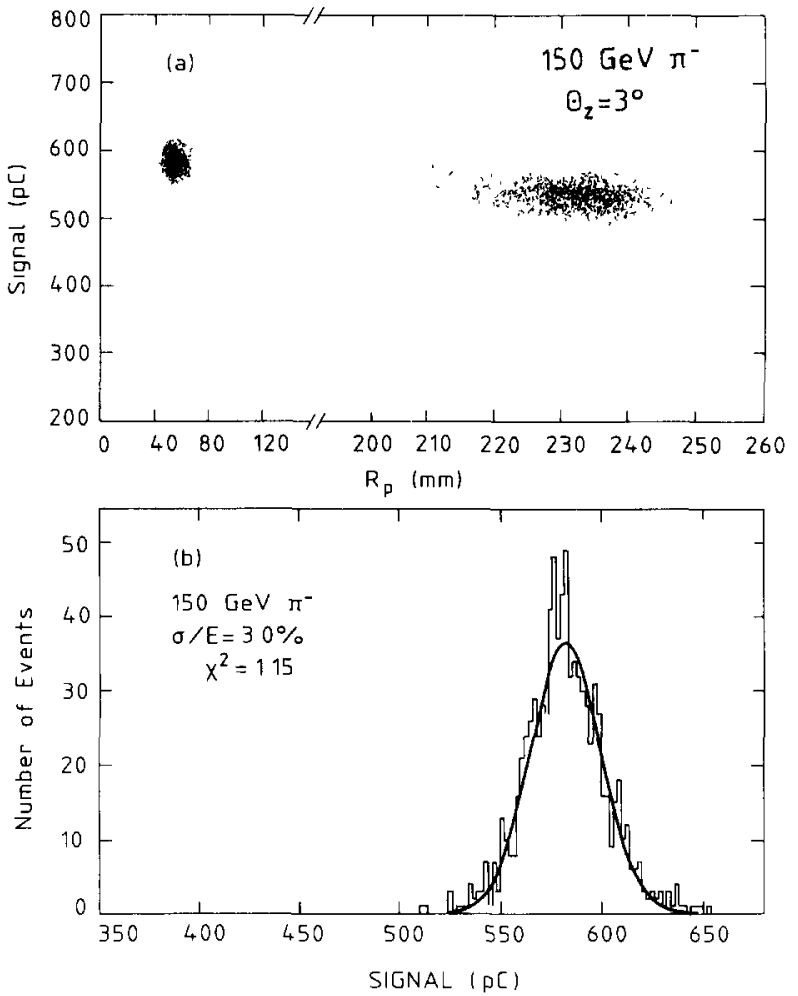

Fig. 20. Scatter plot of the calorimeter signal for $150 \mathrm{GeV}$ particles vs the effective width $R_{\mathrm{p}}$ of the shower (a). The signal distribution for $150 \mathrm{GeV}$ pions after a weighting factor, correcting for the effect of the shower width, has been applied (b). See text for details.

tion for $80 \mathrm{GeV}$ pions improved by $\sim 5 \%$ if the calibration constant for this tower was reduced by $\sim 15 \%$ with respect to the value established on the basis of the response to electron showers. For $9.7 \mathrm{GeV}$ pions the improvement amounted to at most $1 \%$, for a reduction of $10 \%$ of the calibration constant.

Most likely, these phenomena are a consequence of the fact that the calorimeter is slightly undercompensating (see section 5.3). By reducing the calibration constant for the central tower, where most of the $\pi^{0}$ production is detected, one reduces the effect of fluctuations in the $\pi^{0}$ content of the showers on the energy resolution. Evidence for this assumption comes from fig. 20 , where this phenomenon is dealt with in a more sophisticated manner. We defined a quantity $R_{\mathrm{p}}$ as

$R_{\mathrm{p}}=\frac{\sum_{\imath} r_{l} E_{l}^{04}}{\sum_{l} E_{t}^{04}}$,

where $E_{l}$ is the energy deposited in an individual tower $i$ and $r$ is the distance between the center of tower $i$ and the center of gravity of the shower energy deposit profile. This quantity is a measure for the effective width of the shower. It was developed for $\mathrm{e} / \pi$ separation purposes [10]. Fig. 20a shows the total calorimeter signal versus $R_{\mathrm{p}}$. When the shower is wider (i.e. contains less $\pi^{0}$ energy), the signal is somewhat smaller. By weighting the calorimeter signals with an $R_{\mathrm{p}}$ dependent factor, in a way similar to figs. 15 and 17 , the energy resolution could be improved. Moreover, the signal distribution became almost perfectly Gaussian (see fig. 20b, table 3).

It should be emphasized that such procedures are very delicate and mainly of academic interest. They show where the limitations to the energy resolution come from and what the ultimate achievable energy resolutions are. But in practice, one would certainly not want to change the measured calibration constants deliberately by an energy dependent factor (fig. 19). Further improvements in the energy resolution should be pursued by eliminating the causes responsible for the observed effects, rather than by correcting for them.

\subsubsection{Angular dependence}

The angular dependence of the hadronic energy resolution was studied for $40 \mathrm{GeV}$ pions entering the calorimeter at an angle of incidence $\theta_{z}$ ranging from $0^{\circ}$ to $5^{\circ}$. Results, uncorrected for the effects of light attenuation in the fibers, are given in fig. 21 , which shows that, unlike for electrons, the hadronic energy resolution is practically independent of $\theta_{z}$.

The absence of any strong angular dependence may be understood from the fact that the phenomenon that caused the angular dependence for electron detection has no equivalent for pions. Unlike electrons, which lose a large fraction of their initial energy by radiating photons at almost $0^{\circ}$, pions only lose a small fraction of their energy through ionization before undergoing a nuclear interaction in which the secondary particles are

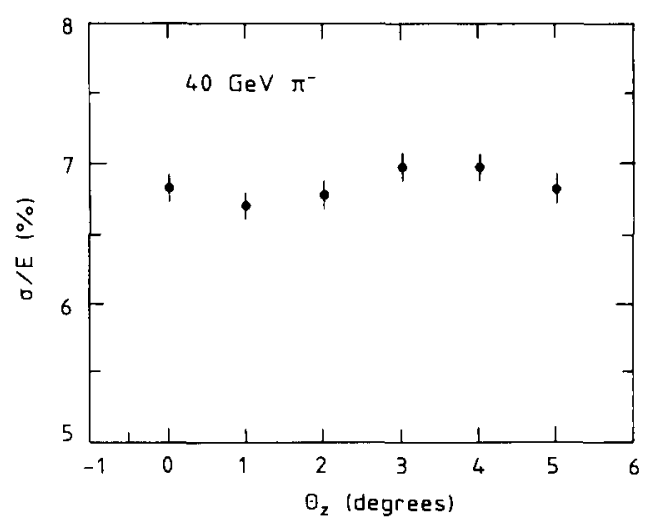

Fig. 21. The energy resolution for $40 \mathrm{GeV} \pi^{-}$detection as a function of the angle of incidence $\theta_{z}$. 


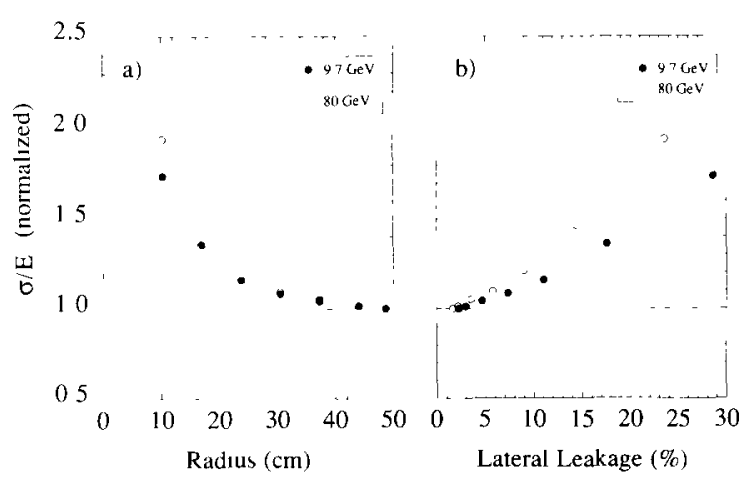

Fig. 22. The hadronic energy resolution as a function of the effective radius of the area over which the calorimeter signal is integrated. The energy resolution is normalized to the value for the whole detector. Results for 9.7 and $80 \mathrm{GeV} \pi^{-}$at $\theta_{z}=3^{\circ}$ (a). The same data as a function of the lateral shower leakage fraction (b).

dispersed over an area that is large compared to the fiber-to-fiber distance. We did not observe any effect of the type shown in fig. 12, which was responsible for the (angle dependent) constant term contributing to the e.m. energy resolution.

\subsubsection{The energy resolution as a function of the cluster} size

In the results shown so far in this section, the hadronic calorimeter signals were obtained by integrating over the total detector volume, i.e. by summing the signals of all individual towers. The lateral cross section of one tower being $48.7 \mathrm{~cm}^{2}$, this corresponds to a cylinder with an effective radius $r$ of $\sqrt{155 \times 48.7 / \pi} \sim$ $49 \mathrm{~cm}$. In the practical circumstances of an experiment (triggering, pile-up), one may want to integrate over a smaller area to determine the energy of a particle or a jet, and the question arises to what extent this would affect the precision of the calorimeter measurements.

We studied this question by integrating the calorimeter signal over smaller and smaller detector areas, leaving out one hexagonal ring after the other (see fig. 1b). The effect on the energy resolution is shown in fig. 22 for pions at 9.7 and $80 \mathrm{GeV}$. The resolution $\sigma / E$, relative to the resolution obtained from integration over the full detector volume, is shown as a function of the effective radius $r$ of the (hexagonal) detector area over which the signal was integrated, in fig. 22a. It turns out that the effect on the hadronic energy resolution is marginal down to $r \approx 30 \mathrm{~cm}$. The relative degradation of the energy resolution for further decreasing radii is somewhat stronger for higher energy particles. The same phenomenon is observed in fig. $22 \mathrm{~b}$, where the energy resolution is shown as a function of the lateral shower leakage fraction (see section 5.3). For a given (average) lateral shower containment, the relative degradation of the hadronic en- ergy resolution due to leakage increases with energy. We found that the effect of lateral shower leakage on the hadronic energy resolution is roughly described by $1 / \sqrt[4]{E}$ term, to be added in quadrature to the scaling term:

$\sigma / E=\sqrt{\left(\frac{c_{1}}{\sqrt{E}}\right)^{2}+\left(\frac{x}{\sqrt[4]{E}}\right)^{2}}+c_{2}$,

where $c_{1}$ and $c_{2}$ are the constants given in eq. (3) and $x$ is the average lateral leakage fraction. An even better fit to the experimental results was obtained when the $x / \sqrt[4]{E}$ term was replaced by a term of the form $A x E^{-b}$. Fitting all data in the energy range 5-150 $\mathrm{GeV}$ simultaneously, we found the best results for $A=0.90$ and $b=0.22$. When the leakage term was added quadratically to the total measured energy resolution rather than to the scaling term, the best fit was obtained for $A=0.87$ and $b=0.17$.

\subsection{The e / $\pi$ signal ratio}

Before entering into the important compensation question, it should be pointed out that there are two issues that should be clearly distinguished:

a) The e/ $\pi$ ratio as derived from the signals actually delivered by the calorimeter. This ratio, which can be directly determined by comparing the signals from electrons and pions at the same energy, is important for the triggering function of the calorimeter in an experiment. It will be a major advantage if the calorimeter responds with signals that, irrespective of the type of particle that caused them, are a direct measure of the energy involved. In practice, one would also like the calorimeter to meet this condition for jets. However, the e/jet signal ratio is not only determined by the properties of the calorimeter, but also by the jet definition. Some particles produced in the fragmentation of a quark or gluon will inevitably pass outside the area defined to contain the jet. The e/jet signal ratio is discussed in section 6.3.

Because of the different longitudinal shower development for electrons, hadrons and jets, the $\mathrm{e} / \pi$ and $\mathrm{e} / \mathrm{jet}$ signal ratios depend on the attenuation characteristics of the fibers.

b) A quantity that we shall call $\mathrm{e} / \mathrm{h}$, which in this context is defined as the ratio of the amount of scintillation light per $\mathrm{GeV}$ of deposited energy produced by the e.m. and non-e.m. components of hadronic showers. This quantity is an intrinsic property of the detector. The contribution of the (large and non-Gaussian) fluctuations in the fraction of e.m. energy generated in hadronic showers to the hadronic energy resolution vanishes only if $e / h=1$. The determination of $e / h$ is the subject of the next subsection. 
Table 4

The e/ $\pi$ signal ratios measured with a spaghetti calorimeter with an effective radius of $49 \mathrm{~cm}$, preceded by a $1.7 X_{0}$ thick preshower detector.

\begin{tabular}{rccl}
\hline$E_{\pi}[\mathrm{GeV}]$ & $\langle$ signal e $\rangle[\mathrm{pC}]$ & $\langle$ signal $\pi\rangle[\mathrm{pC}]$ & $\mathrm{e} / \pi$ \\
\hline 5.0 & $18.65 \pm 0.05$ & $16.99 \pm 0.20$ & $1.097 \pm 0.013$ \\
9.7 & $36.73 \pm 0.04$ & $34.09 \pm 0.07$ & $1.077 \pm 0.003$ \\
20.0 & $78.90 \pm 0.09$ & $73.25 \pm 0.14$ & $1.077 \pm 0.002$ \\
40.0 & $158.9 \pm 0.1$ & $151.6 \pm 0.2$ & $1.048 \pm 0.002$ \\
80.0 & $317.8 \pm 0.2$ & $309.2 \pm 0.4$ & $1.028 \pm 0.001$ \\
150.0 & $579.2 \pm 0.4$ & $593.4 \pm 0.6$ & $0.976 \pm 0.001$ \\
\hline
\end{tabular}

The values of the $\mathrm{e} / \pi$ signal ratio as measured with our detector system are listed in table 4 . The numbers apply to the signals observed in the calorimeter (tables 1 and 3 ), with a $1.7 X_{0}$ thick preshower detector installed upstream. The signals were integrated over an area with an effective radius of $49 \mathrm{~cm}$. The $\mathrm{e} / \pi$ ratio is plotted against the particle energy in fig. 23 . Whereas the errors given in table 4 are statistical only, a systematic error of $1 \%$, added in quadrature, was included in the points shown in fig. 23. This error accounts for instabilities in the PM response (which were never observed to be larger than $0.5 \%$ ), for uncertainties in the beam energies and for the non-Gaussian calorimeter response, which leads to small differences between the mean signal and the mean value of the Gaussian fit.

As for the hadronic energy, we studied the effect of a reduction of the area over which the signals were integrated on the $e / \pi$ signal ratio. The results are shown in fig. 24. As for the hadronic energy resolution, the $\mathrm{e} / \pi$ signal ratio is only marginally affected down to $r \approx 30 \mathrm{~cm}$. Since electrons are already almost fully contained in a cylinder with $r=10 \mathrm{~cm}$, the fact that $\mathrm{e} / \pi$ rises faster at decreasing $r$ if the energy is lower means that the hadronic containment in a cylinder with given radius is increasing with energy.

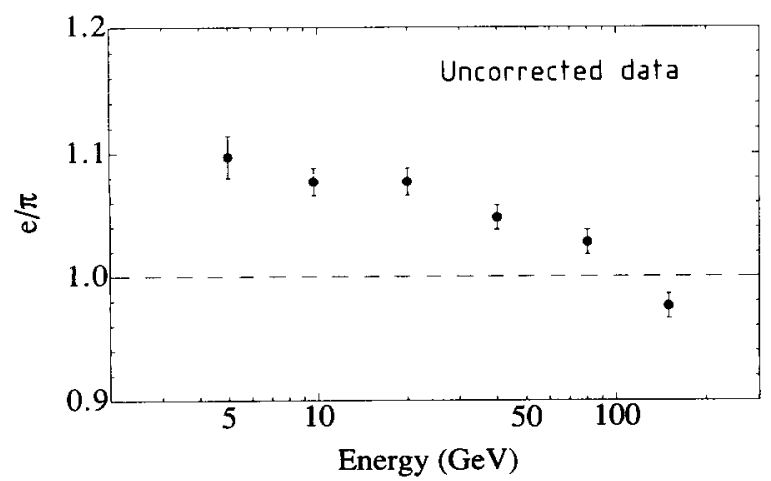

Fig. 23. The e/ $\pi$ signal ratio for the Spaghetti Calorimeter as a function of the particle energy. See text for details.

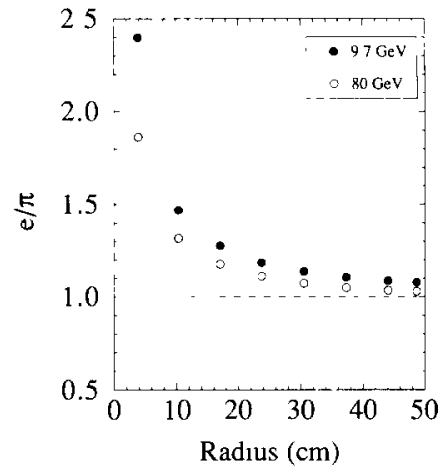

Fig. 24. The $\mathrm{e} / \pi$ signal ratio as a function of the effective radius of the area over which the calorimeter signals are integrated. Results for 9.7 and $80 \mathrm{GeV}$ particles entering the calorimeter at $\theta_{z}=3^{\circ}$.

In earlier tests with the 20-tower prototype of the SPACAL detector, we removed the yellow filters intended to absorb the most strongly attenuated blue light component [9]. Since the light attenuation curve becomes then steeper, and since pion showers develop closer to the PM, the pion signals were more enhanced with respect to the yellow-filter ones than the electron signals. This had the effect of decreasing the measured $\mathrm{e} / \pi$ signal ratio by $7 \%$ at $40 \mathrm{GeV}$. This illustrates that the $e / \pi$ signal ratio is not an intrinsic property of this detector, but can be tuned to a certain extent through the light attenuation characteristics of the fibers. As was pointed out elsewhere [17], radiation damage will also tend to decrease the $e / \pi$ signal ratio. Because of these effects, one might imagine a detector being designed in such a way that eventual effects of radiation damage on the $e / \pi$ signal ratio could be compensated for with filters.

\subsection{The $e / h$ ratio}

In this subsection, we will try to find out to what extent the compensation condition is met by our detector. That is, we will try to determine the relation between the amounts of scintillation light per $\mathrm{GeV}$ of deposited energy for the e.m. and non-e.m. components of hadronic showers. The starting point for this analysis is the $\mathrm{e} / \pi$ signal ratio measured as a function of energy (table 4). In order to determine $e / h$, we have to account for the following effects that have a different influence on the electron and pion signals: a) light attenuation in the fibers, b) energy loss in the preshower detector, c) suppression of very small signals through the low-energy cutoff, d) energy leaking out of the detector.

In table 5 , the differences between the $\mathrm{e} / \pi$ signal ratios that would have been measured in the absence of these instrumental effects and the actually measured 
$\mathrm{e} / \pi$ signal ratios are listed. By combining all these numbers, the $e / \pi$ ratios for this "perfect" detector can be inferred.

a) Light attenuation in the fibers reduces the electron signal more than the hadronic signal and, therefore, it will lead to an underestimation of the $e / \pi$ ratio. Since we know both the effective depth at which the light was produced (fig. 17) and the light attenuation curve of the fibers (fig. 16), the contribution of this effect could be accurately calculated. It varied between $4.2 \%$ at $5 \mathrm{GeV}$ to $5.7 \%$ at $150 \mathrm{GeV}$, the increase with energy stemming from the fact that hadronic showers develop on average deeper inside the calorimeter at higher energies.

b) Energy loss in the preshower detector leads to an effect of the same sign, since it reduces the electron signal more than the hadronic signal. The effects on the electron signals were already quantitatively determined in section 4.2. Pions were assumed to behave like minimum ionizing particles and lost, therefore, only $15 \mathrm{MeV}$ on average in this detector.

c) As was pointed out in section 2.1, signals smaller than 4 ADC counts above the pedestal value were ignored by the readout system. Since the hadronic showers spread over a much larger area than the e.m. ones, neglecting the many small contributions to the calorimeter signal will affect the hadronic response more than the e.m. one. The result is an overestimation of the $\mathrm{e} / \pi$ signal ratio, especially at low energy. In order to estimate the contribution of the signals below threshold to the total calorimeter signal, two methods were employed. In the first method, we studied the effect of a further off-line increase of the cutoff value. Results are given in fig. 25 . One sees that an increase of the cutoff value from 4 counts to, for example, 60 counts above pedestal reduced the 150 $\mathrm{GeV} \pi^{-}$signal by $3 \%$. Assuming that shower shapes are energy independent, a $10 \mathrm{GeV}$ shower can be modelled by a $150 \mathrm{GeV}$ shower where all energies have been scaled by a factor 15 . This means that the 9.7 $\mathrm{GeV}$ pion response would have increased by $3 \%$ if the data acquisition system were 15 times as sensitive. A similar conclusion was reached by studying the effects

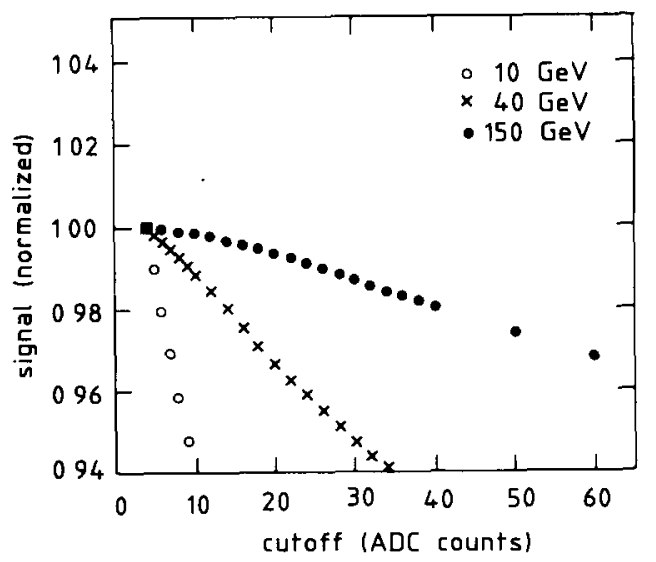

F1g. 25. The average hadronic signal as a function of the low-energy cutoff value, given in counts above pedestal, for pions of $9.7,40$ and $150 \mathrm{GeV}$.

of raising the cutoff values on the 80 and $40 \mathrm{GeV}$ signals. In the second method, the signal distribution was studied tower by tower. Fig. 26 shows a signal distribution that is not affected by this cutoff (fig. 26a) and one that is affected (fig. 26b). In the latter distribution all events below the cutoff threshold end up in the 0 -bin. The data were fit to a Poisson distribution, using the contents of the 0-bin and the observed tail of the distribution as boundary conditions. The resulting curve is also shown in fig. 26b. This procedure was repeated for each tower, and in this way the average total missing signal could be determined. The results obtained with both methods agreed within $10 \%$, the error attributed to the correction for this effect. The effects of the low-energy cutoff on the electron signals were studied as well. As may be expected, the largest contributions from unobserved signals occurred at low energy. At $5 \mathrm{GeV}$, the unobserved component of the signal was found to be $10 \%$ for pions and $4 \%$ for electrons, resulting in a $6 \%$ net effect on the e $/ \pi$ ratio. At $9.7 \mathrm{GeV}$, these numbers were $3 \%$ for pions and $1 \%$ for electrons. At higher energies, the net effect was well below $1 \%$.

The correctness of this procedure was confirmed

Table 5

Influence of instrumental effects on the measured $e / \pi$ signal ratio. Listed are differences between the $e / \pi$ signal ratio that would have been measured in the absence of these effects $(e / \pi$ corrected) and the actually measured $e / \pi$ signal ratio.

\begin{tabular}{cllllll}
\hline$E_{\pi}[\mathrm{GeV}]$ & $\mathrm{e} / \pi$ measured & Light attenuation [\%] & PSD [\%] & Low- $E$ cutoff [\%] & Leakage [\%] & $\mathrm{e} / \pi$ corrected \\
\hline 5.0 & 1.097 & $+4.22 \pm 0.28$ & $+4.72 \pm 0.47$ & $-5.96 \pm 0.60$ & $-2.59 \pm 0.26$ & $1.101 \pm 0.017$ \\
9.7 & 1.077 & $+4.13 \pm 0.20$ & $+3.07 \pm 0.31$ & $-2.13 \pm 0.21$ & $-2.32 \pm 0.23$ & $1.107 \pm 0.006$ \\
20.0 & 1.077 & $+4.01 \pm 0.20$ & $+0.83 \pm 0.08$ & $-0.40 \pm 0.04$ & $-2.13 \pm 0.21$ & $1.102 \pm 0.004$ \\
40.0 & 1.048 & $+4.64 \pm 0.22$ & $+0.55 \pm 0.06$ & $-0.13 \pm 0.01$ & $-1.82 \pm 0.18$ & $1.082 \pm 0.004$ \\
80.0 & 1.028 & $+5.55 \pm 0.24$ & $+0.34 \pm 0.03$ & $-0.05 \pm 0.01$ & $-1.68 \pm 0.17$ & $1.071 \pm 0.003$ \\
150.0 & 0.976 & $+5.67 \pm 0.23$ & $+0.20 \pm 0.02$ & $+0.02 \pm 0.01$ & $-1.50 \pm 0.15$ & $1.056 \pm 0.003$ \\
\hline
\end{tabular}

\footnotetext{
${ }^{a}$ A correction of $+3.6 \%$ was made in order to force linearity for electrons (see text).
} 
independently by the experimental data itself. The ring sum signals, obtained by hardware adding of all the (small) calorimeter signals from towers in the same hexagonal ring (see section 2.1, fig. 2) are much less sensitive to the effects of low-energy cutoff. When determining the particle response using the ring sum signals instead of the software sums of all the individual tower signals, the effects mentioned above were clearly observed. For example, the pion response increased by $8.9 \%$ at $5 \mathrm{GeV}$ and by $2.6 \%$ at $9.7 \mathrm{GeV}$. At high energies ( 80 and $150 \mathrm{GeV}$ ) there remained small $(\sim 0.5 \%)$ differences between the two results, which are most likely due to the fact that the ring signals are the sum of a large number of signals with slightly different calibration constants. This is the reason why

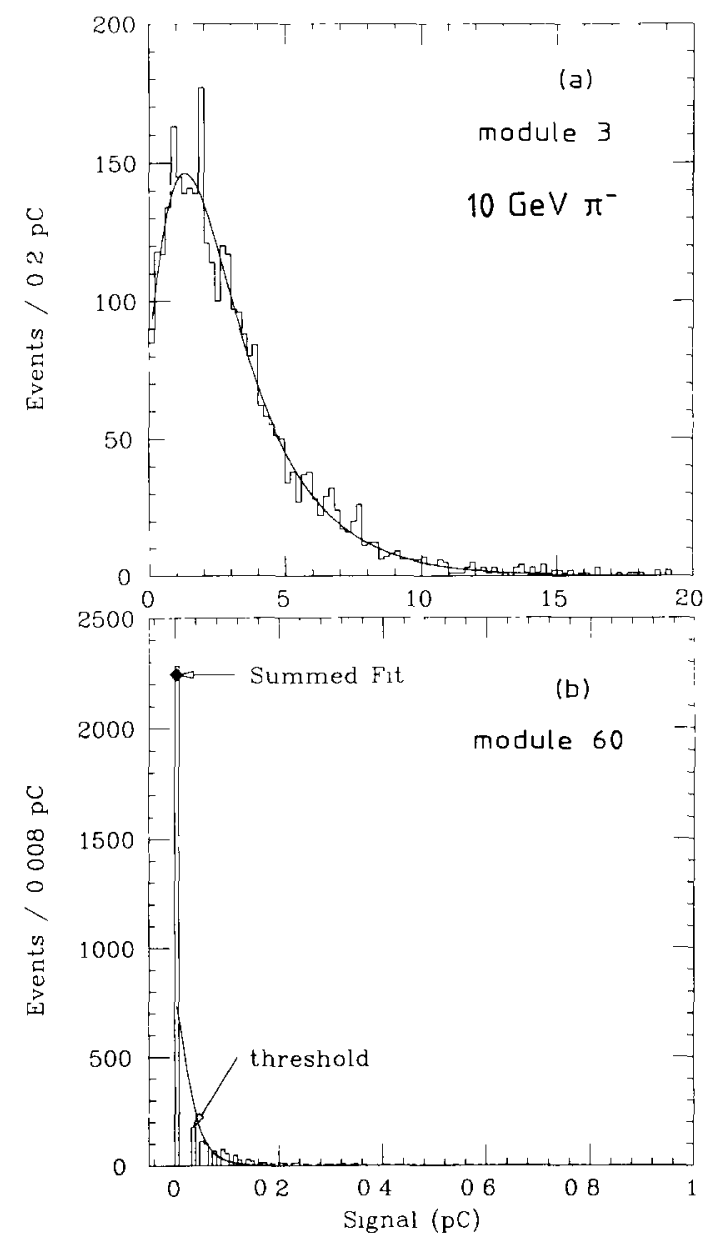

Fig. 26. Typical signal distributions in towers at different distances from the shower axis. A signal distribution that is not affected by the low-energy cutoff at 4 counts above pedestal (a), and a signal distribution that is affected, together with the result of the fit accounting for this effect (b). Data for $10 \mathrm{GeV} \pi^{-}$at $\theta_{z}=3^{\circ}$.

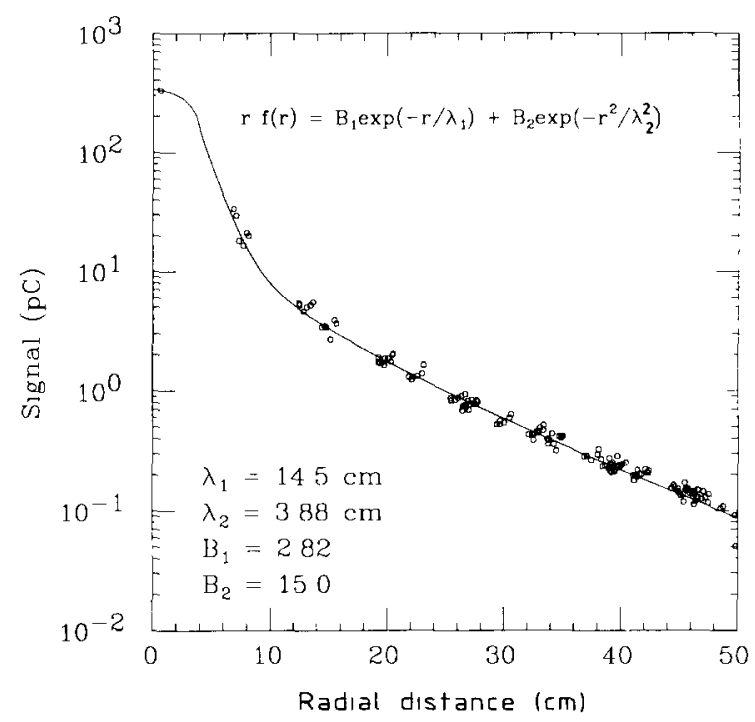

Fig. 27. Lateral shower profile for $150 \mathrm{GeV}$ pions enternng the detector at $\theta_{z}=3^{\circ}$. The average energy deposited per unit surface (i.e. per calorimeter tower) is plotted as a function of the distance between the tower center and the centroid of the shower. The curve is in fact a set of points obtained by integratıng the function $f(r)$, which describes the radial shower profile, over the surface of each tower (see text).

we did not use these ring signals as a basis for our analysis.

d) Energy leakage out of the calorimeter affects the $\mathrm{e} / \pi$ ratio in the same way as the previous effect. Since only hadronic showers were subject to some leakage, the measured $e / \pi$ signal ratio is an overestimation. The leakage consists of two components, a longitudinal and a lateral one. The longitudinal shower leakage is analyzed in another paper [12] and ranges from $0.1 \%$ at $10 \mathrm{GeV}$ to $0.4 \%$ at $150 \mathrm{GeV}$. The lateral leakage was determined from the radial shower profiles. A typical shower profile is given in fig. 27. The experimental points in this figure represent the average signals, for $150 \mathrm{GeV}$ pion showers at $\theta_{z}=3^{\circ}$, measured in each individual tower, as a function of the distance between the tower center and the center of gravity of the shower. The signals were corrected for the effects of low-energy cutoff, which at this energy were very small. The shower profiles are well described by an expression of the following type:

$f(r)=B_{1} / r \exp \left(-r / \lambda_{1}\right)+B_{2} / r \exp \left(-r^{2} / \lambda_{2}^{2}\right)$

This formula represents the differential energy deposit, i.e. $f(r) \mathrm{d} A$ is the energy deposited in an infinitesimal area $\mathrm{d} A=r \mathrm{~d} r \mathrm{~d} \theta$, located at a position $(r, \theta)$ with respect to the shower centroid. In order to compare to the experimental data, $f(r)$ has to be integrated over the (hexagonal) surface of each tower. The curve shown 
in fig. 27 is the result of this procedure. By integrating $f(r)$ from $r=49 \mathrm{~cm}$ to infinity, the lateral shower leakage is obtained. The result is of course most sensitive to the value of $\lambda_{1}$. At low energies, the corrections for low-energy cutoff on the signals in the outer rings were quite substantial, which means that the value of $\lambda_{1}$ is strongly affected by these corrections. Therefore, the value of $\lambda_{1}$ was fixed at $14.5 \mathrm{~cm}$, the value obtained from a free fit for $150 \mathrm{GeV}$ pions, at which energy the parameters could be most precisely determined. The leakage fraction determined in this way ranged from $2.6 \%$ at $5 \mathrm{GeV}$ to $1.5 \%$ at $150 \mathrm{GeV}$. Since the production of $\pi^{0}$ 's in the shower development increases with energy, the $150 \mathrm{GeV} \pi^{-}$showers are better contained laterally than the low-energy ones.

Fig. 27 also offers the possibility to get a realistic feeling for the precision of the calibration constants used in this analysis. Due to fiber-to-fiber response fluctuations (see e.g. fig. 8), PM instabilities, etc. the tower-to-tower calibration constants are definitely much less precise than indicated by the $0.3 \%$ statistical error (see section 3). The fluctuations of the experimental points about the curve in fig. 27 are a measure for these systematic effects. We analyzed these fluctuations and found that the tower-to-tower calibration accuracy is compatible with the results obtained from the grid scan (section 4.1, fig. 10). However, these systematic uncertainties in the tower-to-tower calibration constants turned out to have very little influence on the pion signals, probably because these are usually shared by many towers. The average pion response measured in the 49 points of the matrix scan (section 3) had a distribution with a width of only $\sim 1 \%$. And since the electron signals measured in the center of the calorimeter were very representative for the detector as a whole (see fig. 10), the possible systematic effect on the measured $\mathrm{e} / \pi$ signal ratios is of the same order.

After performing the corrections resulting from a) d), we studied the linearity for electron and pion detection. The results are given in figs. 28 and 29 , respectively. In figs. $28 \mathrm{a}$ and $29 \mathrm{a}$, the average signal per $\mathrm{GeV}$ is given as a function of the energy, for the raw data (the third column in tables 1 and 3 ). These results are of course specific for the setup in which they were obtained, requiring for example a PSD signal larger than $7 \mathrm{mip}$, etc. The results obtained after correcting for the instrumental effects mentioned above are shown in figs. $28 \mathrm{~b}$ and $29 \mathrm{~b}$. It turns out that the alinearity that was at first observed for low-energy electrons (table 1) is completely explained by the effects of energy loss in the PSD and low-energy cutoff. After the corrections were applied, the electron signals were found to be linear within the experimental uncertainties on the beam energy, thus confirming the results of previous measurements performed without the
Linearity to Electrons

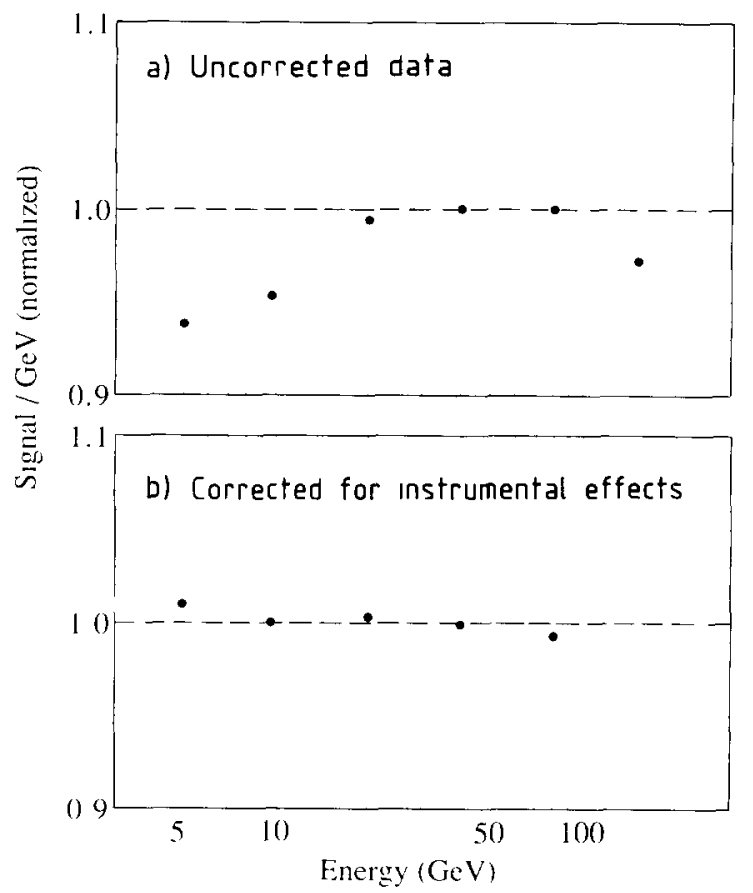

Fig. 28. Signal linearity for e.m. shower detection. The signal per $\mathrm{GeV}$ as a function of energy, for the raw data (a) and after correcting for instrumental effects (b).

Linearity to Pions

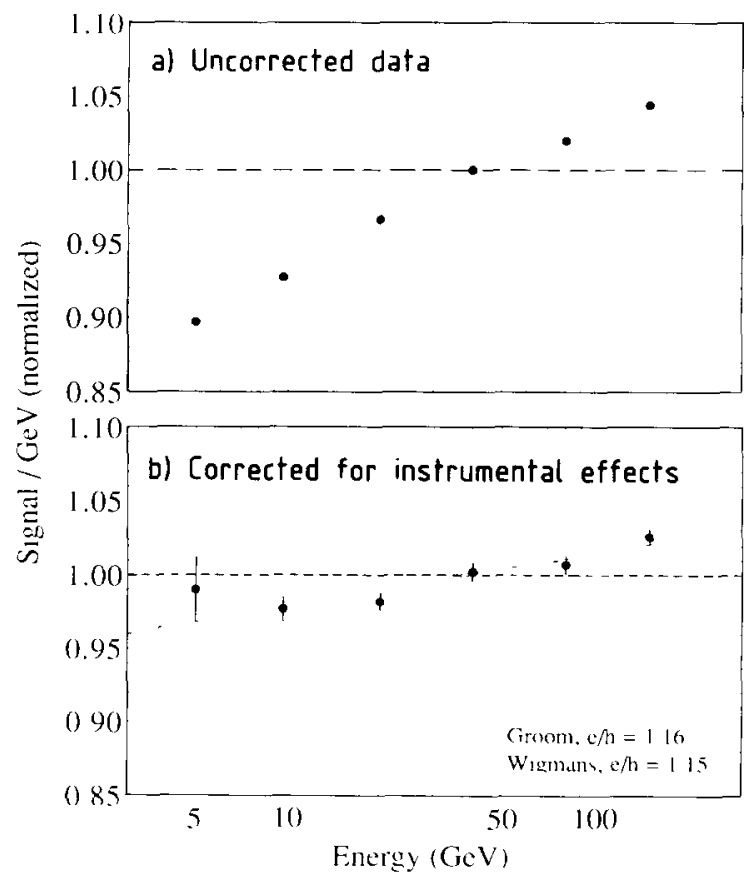

Fig. 29. Signal linearity for pion detection. The signal per $\mathrm{GeV}$ as a function of energy, for the raw data (a) and after correcting for instrumental effects (b). The curves correspond to fits for $\mathrm{e} / \mathrm{h}=1.15-1.16$ (see text for details). 


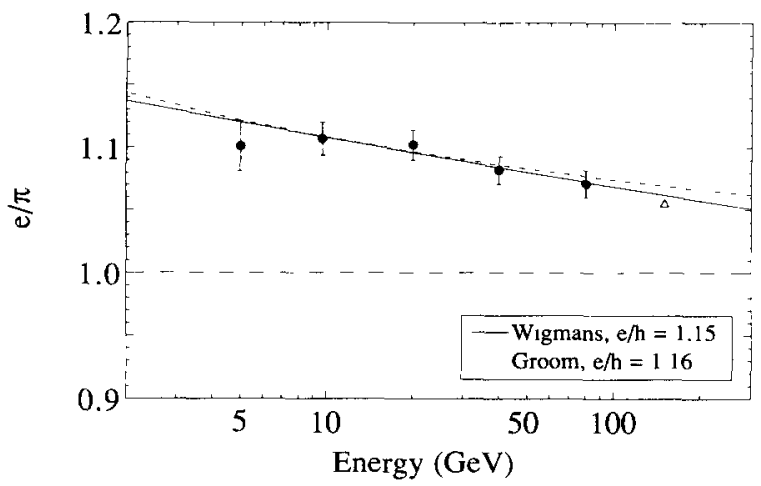

Fig. 30. The $e / \pi$ ratio corrected for instrumental effects as a function of energy. The curves corresponds to fits for $\mathrm{e} / \mathrm{h}=$ $1.15,1.16$. The point at $150 \mathrm{GeV}$ was not included in the fits. See text for details.

preshower detector [9]. On the other hand, the signal at $150 \mathrm{GeV}$ was significantly too low, by about $3.6 \%$. This was not observed in the measurements before. We suspect that this phenomenon was caused by saturation of the PM tube, due to space charge effects. The PM gain in these measurements was a factor four larger than in the ones that showed perfect linearity up to 150 $\mathrm{GeV}$ [9], because of the splitting of the anode signals (section 2.1). This effect did not play a role for the 150 $\mathrm{GeV}$ pion signals, since the central tower detected on average only half the energy (equivalent to the $80 \mathrm{GeV}$ electron signals) in this case. Fig. 29 shows that the detector is slightly alinear for the detection of pion signals. Over the energy range $5-150 \mathrm{GeV}$, the signal per $\mathrm{GeV}$ increased by $\sim 5 \%$.

In order to correct for the PM saturation, the e $/ \pi$ ratio for $150 \mathrm{GeV}$ was increased by $3.6 \%$, thus forcing linearity for e.m. shower detection. The resulting $e / \pi$ values, corrected for instrumental imperfections, are shown as a function of energy in fig. 30 . As in the case of the uncorrected $\mathrm{e} / \pi$ values, the error bars in this figure include a systematic error of $1 \%$, added in quadrature to the values listed in table 5 .

All that we need in addition to be able to determine the $\mathrm{e} / \mathrm{h}$ value, is the average fraction of e.m. energy $\left(f_{\pi^{0}}\right)$ produced in pion showers. The relation between $\mathrm{e} / \mathrm{h}$ and the $\mathrm{e} / \pi$ signal ratios as a function of energy was investigated by Wigmans [3] and by Groom [18], who assumed for $f_{\pi^{0}}(E)$ a logarithmic and a power-law dependence, respectively. The authors gave the following formulae, which globally describe experimental data:

Wigmans:

$\mathrm{e} / \pi(E)=\frac{\mathrm{e} / \mathrm{h}}{1-(1-\mathrm{e} / \mathrm{h}) k \ln E}$

with $k=0.11$;
Groom:

$$
\begin{gathered}
\mathrm{e} / \pi(E)=\frac{1}{1-(1-\mathrm{h} / \mathrm{e})\left(E / E_{0}\right)^{m-1}} \\
\left(m=0.85, E_{0}=1 \mathrm{GeV}\right) .
\end{gathered}
$$

Using these expressions, our data is best fit by the value

$\mathrm{e} / \mathrm{h}=1.15 \pm 0.02$.

The curves in fig. 30 are the results of best fits of eqs. (8a) and (8b) to our experimental data. The $150 \mathrm{GeV}$ point was not included in these fits, but is reasonably well described by the resulting curves. The same formalisms also describe the observed signal alinearity for pion detection well, as illustrated by the curves in fig. 29.

\section{Multiparticle detection}

\subsection{Energy resolution}

In an attempt to simulate jet detection with this calorimeter, we measured the reaction products of

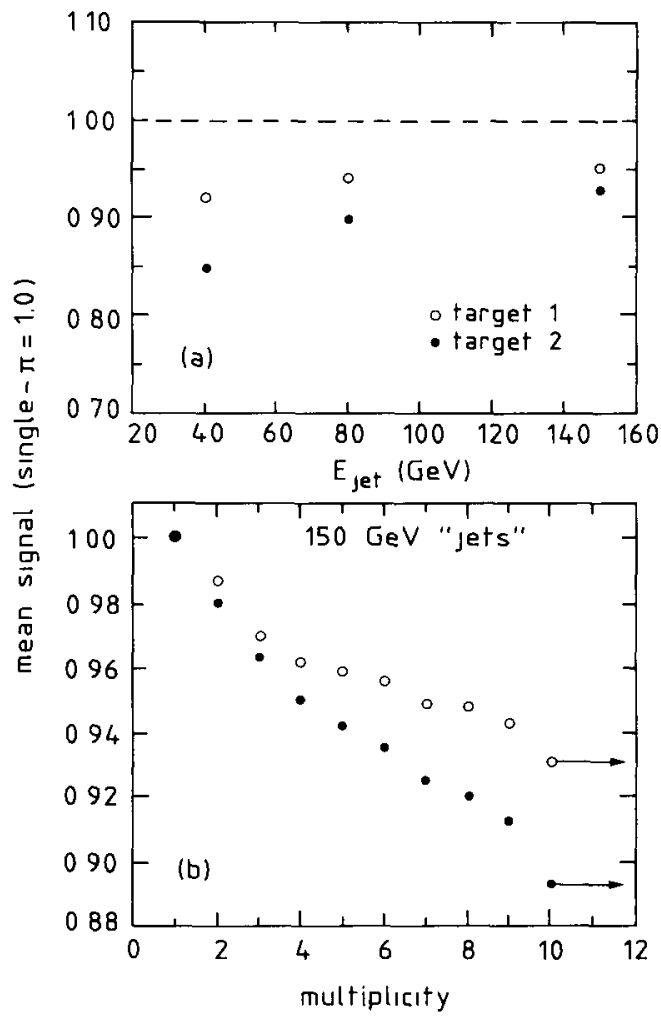

Fig. 31. Multiparticle signals, integrated over the total calorimeter, as a function of energy (a) and as a function of the multiplicity of the interaction (b). Interactions with a multiplicity $\geq 10$ were grouped together in the last bin. 
pions interacting in a block of paraffin installed either $44 \mathrm{~cm}$ (position 1) or $147 \mathrm{~cm}$ (position 2) upstream from the front face of the calorimeter. Interactions in the target were selected and triggered upon using the signal from the $S_{\mathrm{J}}$ counter (see fig. 3). The trigger threshold was set at twice the minimum ionizing value. These measurements were performed at 40,80 and 150 GeV.

One of the problems in interpreting the measurement results comes from the fact that, unlike for single particles, the energy deposited in the calorimeter was not precisely known. In the interaction in the $(10 \mathrm{~cm}$ thick) target, a certain amount of energy may be spent in breaking up and recoil of the target nucleus, some energy may be absorbed in the paraffin and, finally, secondary particles may have been produced at such large angles that they geometrically missed the calorimeter. And since the fraction of lost energy may wildly fluctuate from event to event, these phenomena will have contributed to the width of the signal distributions measured with the calorimeter. The first conclusion is, therefore, that the energy resolution for jets with precisely known energy will be better than the one measured in this way.

Evidence for the phenomena mentioned above comes from the fact that a) the multiparticle signals were smaller than the ones for single pions, and the difference increased with decreasing energy, b) the signals measured at position 2 were considerably smaller than at position 1 , and $c$ ) the signals became smaller when the multiplicity of the interaction increased.

This is illustrated in fig. 31. In fig. 31a, the multiparticle signals, integrated over the whole detector, are given relative to the single-pion signals as a function of energy. In fig. 31b, the multiparticle signals for one energy $(80 \mathrm{GeV})$ are shown as a function of the multiplicity of the interaction. This multiplicity could be inferred from the signal in the $S_{J}$ counter.

There is also another effect that caused the multiparticle signals to be smaller than the corresponding single-pion ones. On average, one third of the particles produced in the interactions in the upstream target developed e.m. showers $\left(\pi^{0}\right.$ 's) and the other particles were lower in energy than the incoming pions. Therefore, light production in the multiparticle events took place further upstream in the calorimeter than for the single-pion events. The difference in light attenuation caused the multiparticle signals to be smaller. To estimate this effect quantatatively, one may consider the multiparticle events as hadron showers with a fixed starting point, i.e. in the target upstream of the calorimeter. Single pions detected by the calorimetet will have traversed on average $1 \lambda_{\pi}(=31.4 \mathrm{~cm}[10,11])$ of material before starting the shower. From eq. (2), one may then infer that the different light attenuation

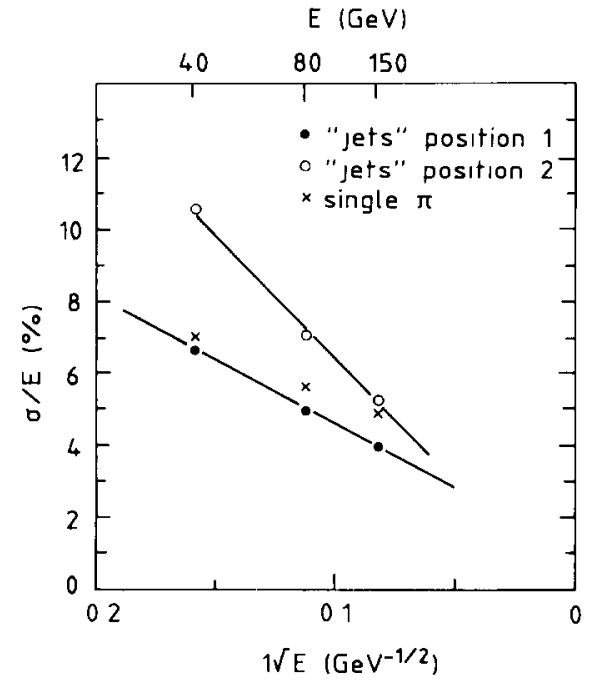

Fig. 32. The widths of the multiparticle signal distributions, measured in positions 1 and 2, compared to the uncorrected single-pion energy resolution.

will cause the multiparticle signals to be $3-4 \%$ smaller than the corresponding single-pion signals. Analysis of the lateral displacement of the center of gravity of the shower with respect to the axis of the incoming "jet" (cf. fig. 17) confirmed that the light production was indeed shifted upstream by $\sim 1 \lambda_{\pi}$ for the multiparticle events.

The 3-4\% signal decrease coming from this phenomenon accounts for most of the effect observed for $150 \mathrm{GeV}$ "jets" produced in target position 1 (see fig. 31a). In other cases, e.g. at lower energy, at high multiplicity, or for interactions in target position 2, the contribution of missing energy to the observed signal decrease may be substantial, up to $\sim 10 \%$. From fig. $22 \mathrm{~b}$ and eq. (6) one may infer the effect of such "leakage" on the width of the signal distributions. But in spite of this, the measured signal distributions looked remarkably good, especially at high energy. In fig. 32, the widths of the signal distributions for the secondaries from interactions at positions 1 and 2 are compared to the (uncorrected) single-pion energy resolutions. In position 1, the multiparticle resolution at 80 and $150 \mathrm{GeV}$ was even significantly better than for single pions. This can be explained from the fact that light attenuation in the fibers, which played a major role in the energy resolution for single pions (section 5.1 , was much less important for multiparticle detection. Since several particles entered the calorimeter simultaneously, the fluctuations in the longitudinal center of gravity of the light production, which caused the energy resolution for single pions to deviate from a pure scaling behavior, are suppressed by a factor depending on the number of particles in the "jet".

We investigated the energy resolution as a function 


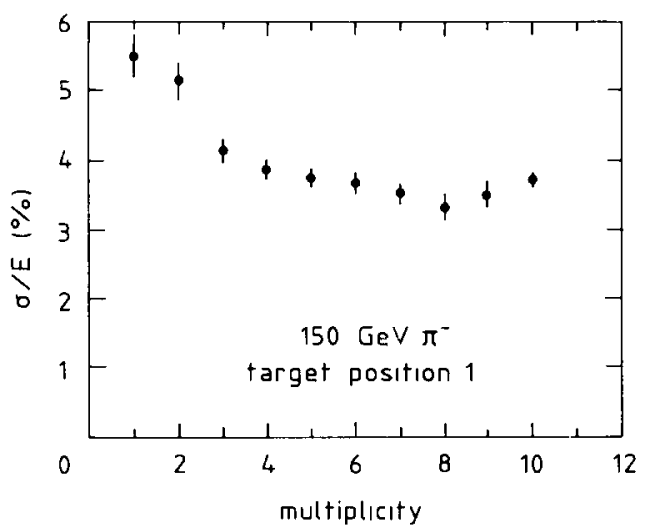

Fig. 33. The multıparticle energy resolution as a function of the interaction multıplicity, for $150 \mathrm{GeV} \pi^{-}$interactıng in position 1 . Interactions with a multiplicity $\geq 10$ were grouped together in the last bin. of the multiplicity and did indeed find a dependence. Fig. 33 shows the multiparticle resolution at $150 \mathrm{GeV}$ as a function of the multiplicity, or rather as a function of the signal in the $S_{J}$ counter. The resolution improves at increasing multiplicity, leveling off and then deteriorating beyond a multiplicity of $\sim 8$. The latter effect is probably due to the fact that fluctuations in the average missing energy start dominating at that point, in agreement with fig. $31 \mathrm{~b}$.

We also found that the multiparticle signal distribution fit a Gaussian curve much better than the (uncorrected) single-pion one. In fig. 34, the $150 \mathrm{GeV}$ singleand multiparticle distributions are compared. Also here, the distributions became "more Gaussian" when the multiplicity increased (fig. 34c).

One problem in the detection of jets in an experiment concerns the definition of the jet, i.e. the detector area over which should be integrated. In practice,
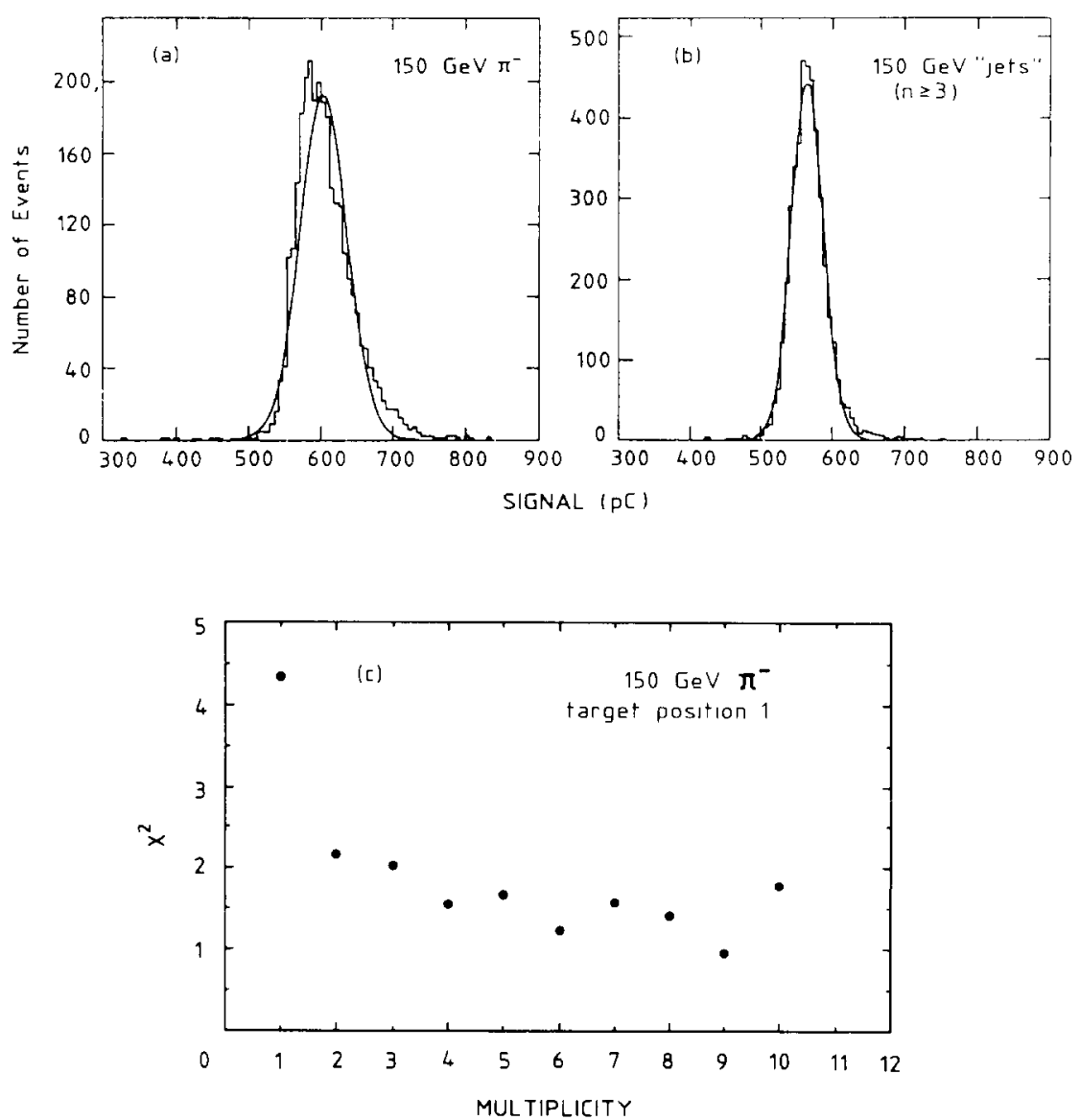

Fig. 34. Signal distributions for $150 \mathrm{GeV} \pi^{-}$(a) and for $150 \mathrm{GeV} \pi^{-}$interacting in position 1 , with a multiplicity $\geq 3$ (b). The $\chi^{2}$ of a Gaussian fit to the signal distribution, as a function of the multiplicity of the interaction (c). Data are for $150 \mathrm{GeV} \pi^{-}$ interacting in positıon 1 . Interactions with a multiplicity $\geq 10$ were grouped together in the last bin. 
one frequently defines a cone with a certain opening angle around the thrust axis for this purpose. However, some soft jet components will almost always be excluded in doing so. In a sense, this situation has some similarity with our multiparticle detection. No matter how big the detector, some fraction of the energy may always escape detection. We studied the width of the multiparticle signal distribution as a function of the opening angle of a cone around the beam axis. Since the particles developed showers over some depth inside the calorimeter, the opening angle was calculated with respect to a plane at a depth of $60 \mathrm{~cm}\left(\sim 2.9 \lambda_{I}\right)$ inside the calorimeter, rather than with respect to the front surface. This depth corresponds to the average center of gravity of $80 \mathrm{GeV}$ pion showers in the calorimeter [10]. The results are given in fig. 35. They show that in this way,the average signal and the width of the multiparticle signal distribution measured in positions 1 and 2 can be related to each other, since they lie both on approximately the same smooth curves. From the curve in fig. $35 \mathrm{~b}$, we conclude that the energy resolution does not improve significantly for opening angles beyond $\sim 45^{\circ}$.

\subsection{The e / jet signal ratio}

In section 5.1 , we argued that the $\mathrm{e} / \pi$ signal ratio is an important quantity for triggering purposes. In prac-

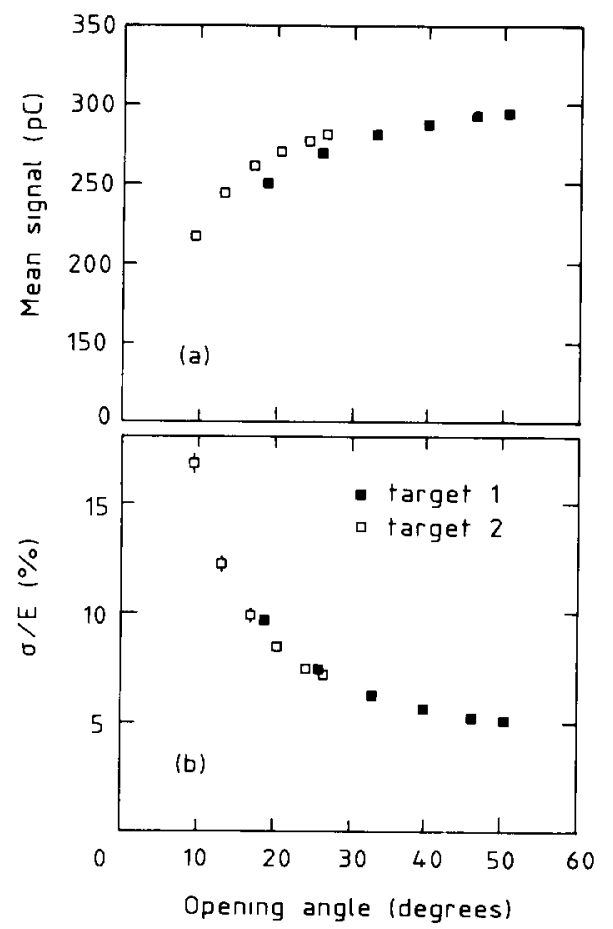

Fig. 35. The average multiparticle signal (a) and the average width of the multiparticle signal distribution (b) as a function of the opening angle of a cone around the beam axis. Data for $80 \mathrm{GeV} \pi^{-}$interacting at positions 1 and 2 .

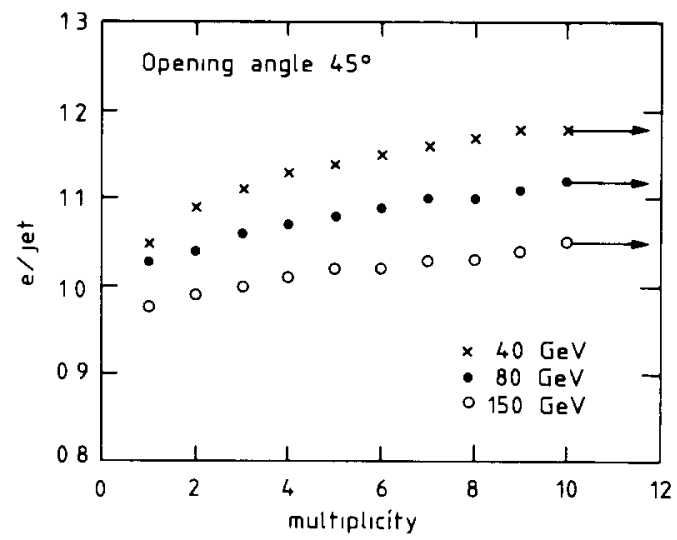

Fig. 36. The e/jet signal ratıos as a function of the multiplicity, at 40,80 and $150 \mathrm{GeV}$. The jet is defined by a cone with an opening angle of $45^{\circ}$. Interactions with a multuplicity $\geq 10$ were grouped together in the last bin.

tice, the e/jet signal ratio is probably equally, if not more, important. The accuracy with which the jet energy can be measured, determines largely the accuracy of the mass measurement of, for example, the top quark.

In the previous subsection, we already gave the arguments why the multiparticle signals are on average smaller than the single-pion signals: differences in light attenuation, missing energy for a given jet definition. As a consequence, the $\mathrm{e} / \mathrm{jet}$ signal ratios are somewhat larger than the $\mathrm{e} / \pi$ signal ratios. In fig. 36 , the e/jet signal ratios are given for a "jet" with an opening angle of $45^{\circ}$, as a function of multiplicity. The values range from 1.00 to 1.05 for $150 \mathrm{GeV}$ jets with a multiplicity of at least three, and from 1.11 to 1.18 for $40 \mathrm{GeV}$ jets. If the opening angle is decreased, the e/jet signal ratios increase further.

\section{Discussion}

\subsection{Compensation}

The result on the $e / h$ ratio (9) shows that the calorimeter is not completely compensating. Other data obtained in this analysis confirm this conclusion, e.g. the signal alinearity for pion detection [3] (fig. 29) and the fact that the width of the signal distributions may be improved by deliberately using the wrong calibration constants (fig. 19). The constant term in the energy resolution left after eliminating the effects of light attenuation, i.e. $1.0 \%$ when added linearly to the scaling term (3b), is in agreement with predictions. In ref. [3], a $1 \%$ constant term, to be added linearly to the scaling term, was predicted for a calorimeter with an $\mathrm{e} / \pi$ signal ratio of 1.1 at $10 \mathrm{GeV}$. 
The question arises of how it is possible that our calorimeter, which has the same sampling fraction as the $\mathrm{Pb}$ /scintillator sandwich calorimeter built by ZEUS [4], has an e/h value that is larger by about $10 \%$. First of all it should be mentioned that the $1.05 \pm 0.04$ value reported by Bernardi et al. [4] was an average $e / \pi$ signal ratio and not an $\mathrm{e} / \mathrm{h}$ value. Moreover, their error bar is quite large compared to the difference that we are talking about, but even so there might be a $2 \sigma$ effect. There are several factors that may contribute to the difference between the $\mathrm{e} / \mathrm{h}$ ratios measured by ZEUS and by us. We mention the most important ones: 1) Our fibers have a plastic cladding with a thickness of $29 \mu \mathrm{m}$. This corresponds to $11 \%$ of the total plastic volume. Neutrons interacting in the plastic cladding will in general not be detected. Our detector is, therefore, less efficient in sampling neutrons than the plate calorimeter. 2) When the distance between subsequent sampling layers is decreased below $\sim 1 X_{0}$, the e/mip ratio tends to increase, as was shown in ref. [3]. Therefore, in our detector the e.m. shower part is more efficiently sampled than in the case of the plate calorimeter.

Both effects work in the same direction, i.e. they increase $\mathrm{e} / \mathrm{h}$. Knowing the precise $\mathrm{e} / \mathrm{h}$ value for this calorimeter is very important, since it makes it possible to predict the effects of changes in the composition of the calorimeter and to design an instrument that is completely compensating to within a few percent. There are several ways in which this goal may be achieved, e.g. 1) the cladding could be made thinner, or its hydrogen content might be reduced, 2) a very thin layer of material with $Z \ll 82$ could be inserted in between the lead and the fiber, thus selectively reducing the e.m. response [19], 3) the sampling fraction could be decreased, for the same sampling frequency, and 4) the sampling frequency could be decreased, for the same sampling fraction, or by a combination of several of these methods.

\subsection{Dynamic range}

Our results clearly demonstrate that if one wants to fully exploit the potential of this type of calorimeter, a light-detection/data-acquisition system with a very large dynamic range is needed. The 4.5 orders of magnitude available to us were insufficient. Our measurements concerned particles in the range of 5-150 $\mathrm{GeV}$, detected with the same gain settings at all energies. This factor 30 is still at least an order of magnitude below the range of energies at which precise calorimetric measurements will be needed in $\mathrm{LHC}$ / SSC experiments. But even for this factor 30 , the shortcomings of our PM/ADC system were apparent (see section 5.3). At the highest energy, signal saturation due to space charge effects in the last acceleration stage of the PM affected the results of the measurements, at the lowest energies pedestal fluctuations in the ADC formed the limit. This is one of the reasons why we are developing a new type of light detector, which is expected to have a larger dynamic range of operation than a photomultiplier [20].

\subsection{Energy resolution and signal uniformity}

Our results show that the fiber quality is the main factor that limits the performance of the calorimeter: 1) Fiber-to-fiber response fluctuations cause a signal nonuniformity for e.m. showers that is larger than the energy resolution beyond $100 \mathrm{GeV}$, and 2) light attenuation in the fibers deteriorates the energy resolution for pions and leads to non-Gaussian response functions, especially at high energy.

The first point is probably more serious than the second one. As was shown in our analysis, light attenuation can be corrected for in the case of single pions and its effects are strongly reduced for jets, especially at high multiplicity.

Because of the small Molière radius, the number of different fibers that effectively contribute to the shower signal is small as well. It was estimated that fiber-tofiber response fluctuations of $\sim 6 \%$ lead to a $1 \%$ nonuniformity for e.m. shower signals [13]. In practice, the fluctuations were a factor 2-3 larger (fig. 10). Further improvement of the fiber quality, and in particular of the reproducibility of the fiber response, is therefore needed.

It should be noted that a more frequent shower sampling would reduce the sensitivity to fiber-to-fiber response fluctuations. If the fiber diameter were reduced to $0.5 \mathrm{~mm}$, the tolerable level of such fluctuations would increase to $12 \%$, if $1 \%$ nonuniformity is the design goal. Such a reduction of the fiber diameter would also improve the e.m. energy resolution. The scaling term is expected to be reduced by a factor $\sqrt{2}$. Based on our understanding of the origin of the constant term (section 4.1), we also expect a considerable reduction of this term. Of course, such an increased sampling frequency would make the detector more difficult to build and also morc expensive.

The longitudinal uniformity would greatly benefit from a suppression of the short component in the light attenuation curve (eq. (2)). Preventing the reflection of light at the interface cladding/air is the recipe for achieving this goal. All these topics require further R \& D.

\section{Summary and conclusions}

We have studied the detection of showering particles with a 13-ton lcad/scintillating-fiber calorimeter 
(SPACAL) designed to be compensating (lead: fiber ratio $4.2: 1$ in volume). The detector roughly had the shape of a $9.6 \lambda$, deep cylinder with a diameter of $4.7 \lambda_{I}$. It was subdivided in 155 longitudinally unsegmented towers, in which the fibers were running (roughly) in the same direction as the incoming particles, such as to achieve the best possible detector hermeticity. The detector signals were studied for particles in the energy range from 5 to $150 \mathrm{GeV}$.

For electrons, the energy resolution $\sigma / E$ was measured to be $12.9 \% / \sqrt{E}$, plus a constant term depending on the angle $\theta_{z}$ between the particle direction and the fiber axis. This term, which amounts to $1.2 \%$ for $\theta_{z}=3^{\circ}$, was shown to be due to anomalous sampling in the early stage of the shower development. It was greatly reduced when only electrons entering the detector in the lead were taken into account. The effect on energy resolution and signal linearity of a $1.7 X_{0}$ thick preshower detector, installed $12 \mathrm{~cm}$ upstream from the detector, was measured to be small. Only for energies below $20 \mathrm{GeV}$ was more than $1 \%$ of the shower energy absorbed by this detector, and its effect on the energy resolutions measured with SPACAL was negligible in all cases. Energy loss in this preshower detector caused some signal alinearity for electrons at low energy, which could be recovered by using the signals from the preshower detector. The e.m. energy resolutions achieved with SPACAL were slightly better than predicted with Monte Carlo simulations.

Single pions were detected with an energy resolution of $\sim 30 \% / \sqrt{E}$ plus a constant term, which turned out to be mainly due to the effects of light attenuation in the fibers. Light attenuation also caused deviations from a Gaussian response function. With knowledge of the impact point of the particles, these effects could be efficiently removed for single pions. The energy resolution for pions was found to be practically independent of the angle $\theta_{z}$. Measurements of the hadronic energy resolution as a function of the cluster size showed that the results obtained for a cylindrical region with a radius of $30 \mathrm{~cm}$ around the particle's impact point were practically equal to the ones obtained for fully contained showers. The $\mathrm{e} / \pi$ signal ratio was measured to be smaller than 1.10 in the energy range from 5 to 150 $\mathrm{GeV}$. When the cluster size was limited to a cylindrical region with a radius of $30 \mathrm{~cm}$ around the particle's impact point, these values increased by $\sim 4 \%$. By removing the yellow filters, which changed the light attenuation characteristics of the fibers, the $\mathrm{e} / \pi$ signal ratios decreased, by $7 \%$ at $40 \mathrm{GeV}$. After removing the instrumental effects, caused by light attenuation, by absorption in the preshower detector, by the insufficient dynamic range of our PM/data acquisition system and by shower leakage, we found the intrinsic $e / h$ value of this detector to be $1.15 \pm 0.02$. The reasons why the compensation goal was not completely achieved are qualitatively understood and several recipes exist for designing completely compensating lead/fiber calorimeters, using our experimental results as a starting point.

The properties for jet detection were studied with particles originating from nuclear interactions in a target upstream from the calorimeter. The effects of light attenuation in the fibers were found to be much less important than for single-pion detection. At increasing multiplicity, the measured signal distributions became more Gaussian and the energy resolution improved. Especially at high energy, the multiparticle resolution was found to be considerably better than the single-pion one. The $\mathrm{e} / \mathrm{jet}$ signal ratios were slightly larger than the $e / \pi$ signal ratios, due to energy leaking out of the region defining the jet and to the increased effect of light attenuation. For a cone with an opening angle of $45^{\circ}$, the e /jet signal ratio was measured to range from 1.18 at $40 \mathrm{GeV}$ to 1.05 at $150 \mathrm{GeV}$ for high-multiplicity “jets".

\section{Acknowledgements}

We are deeply grateful to the LAA Project Leader, Prof. Dr. A. Zichichi, for his vision, his warm interest and his encouraging support. The work described in this paper would have been impossible without the outstanding technical support provided by our technicians O. Barnaba, C. Baudoin, M. Borriello, S. Bricola, J.-M. Chapuis, C. Farella, B. Foligne, A. Freddi, G. Improta, G. Iuvino, F. Pagano, S. Robinson, R. Rocco, L. Rose-Dulcina, G. Sannier, C. Schillinger, A. Sigrist and V. Vanzanella. Financial support from the Istituto Nazionale di Fisica Nucleare to the Cagliari, Napoli and Pavia groups, from the Conselho Nacional de Desenvolvimento Cientifico e Tecnologico of Brazil to the Rio de Janeiro group and from the U.S. Department of Energy and National Science Foundation to the San Diego group is acknowledged. And finally, we are grateful to the staff of the SPS, and in particular to $\mathrm{N}$. Doble, for the excellent beam conditions and assistance provided during our tests.

\section{References}

[1] D.M. Atwood et al., Proc. Workshop on Experıments, Detectors and Experimental Areas for the Supercollider, Berkeley, 1987, eds. R. Donaldson and M.G.D. Gilchriese (World Scientific, Singapore, 1988) p. 728.

[2] C.W. Fabjan and R. Wigmans, Rep. Progr. Phys. 52 (1989) 1519 , and references therein.

[3] R. Wigmans, Nucl. Instr. and Meth. A259 (1987) 389.

[4] E. Bernard et al., Nucl. Instr, and Meth. A262 (1987) 229. 
[5] G. Drews et al., Nucl. Instr. and Meth. A290 (1990) 335.

[6] H. Tiecke, Nucl. Instr. and Meth. A277 (1989) 42.

[7] R. DeSalvo et al., Nucl. Instr. and Meth. A279 (1989) 467.

[8] D. Acosta et al., Nucl. Instr. and Meth. A302 (1991) 36.

[9] D. Acosta et al., Nucl. Instr. and Meth. A294 (1990) 193.

[10] D. Acosta et al., Nucl. Instr. and Meth. A305 (1991) 55.

[11] We define the nuclear mteraction length $\lambda_{I}$ in the same way as the Particle Data Group in Phys. Lett. B239 (1990), page III 5, 6. It is the mean free path for protons between inelastic interactions. We will use the symbol $\lambda_{\pi}$ for pions. Since the inelastic cross sections for protons are $50 \%$ larger than for pions, $\lambda_{\pi} \approx 1.5 \lambda_{I}$.

[12] D. Acosta et al., On Muon Production and other Leakage Aspects of Pion Absorption in a Lead/ScintillatingFiber Calorimeter, preprint CERN-PPE/91-107 (1991), to be published in Nucl. Instr. and Meth.

[13] F.G. Hartjes and R. Wigmans, Nucl. Instr. and Meth. A277 (1989) 379.
[14] A. Andresen et al., Nucl. Instr. and Meth. A290 (1990) 95.

[15] C.M. Hawkes et al., Nucl. Instr. and Meth. A292 (1990) 329.

[16] G.R. Young et al., Nucl. Instr. and Meth. A279 (1989) 503.

[17] D. Acosta et al., The Effects of Radiation Damage on a Scintillating Plastic Fibre Calorimeter, preprint CERNPPE /91-45 (1991), submitted to Nucl. Instr. and Meth.

[18] D.E. Groom, Proc. Workshop on Calorimetry for the Supercollider, Tuscaloosa, 1989, eds. R. Donaldson and M.G.M. Gilchriese, p. 59.

[19] R. Wigmans, Nucl. Instr. and Meth. A265 (1988) 273; E. Borchi et al., Phys. Lett. B222 (1989) 525.

[20] The SPACAL Collaboration, Scintillating Fibre Calorimetry at the LHC, CERN-DRDC/90-23 (1990). 